\title{
Robotic surgery: disruptive innovation or unfulfilled promise? A systematic review and meta-analysis of the first $\mathbf{3 0}$ years
}

\author{
Alan $\operatorname{Tan}^{1} \cdot$ Hutan Ashrafian ${ }^{1} \cdot$ Alasdair J. Scott ${ }^{1} \cdot \operatorname{Sam}_{\text {E. Mason }}{ }^{1} \cdot$ \\ Leanne Harling $^{1}$ - Thanos Athanasiou ${ }^{1}$ - Ara Darzi ${ }^{1,2}$
}

Received: 25 August 2015/Accepted: 11 January 2016/Published online: 19 February 2016

(C) The Author(s) 2016. This article is published with open access at Springerlink.com

\begin{abstract}
Background Robotic surgery has been in existence for 30 years. This study aimed to evaluate the overall perioperative outcomes of robotic surgery compared with open surgery (OS) and conventional minimally invasive surgery (MIS) across various surgical procedures.

Methods MEDLINE, EMBASE, PsycINFO, and ClinicalTrials.gov were searched from 1990 up to October 2013 with no language restriction. Relevant review articles were hand-searched for remaining studies. Randomised controlled trials (RCTs) and prospective comparative studies (PROs) on perioperative outcomes, regardless of patient age and sex, were included. Primary outcomes were blood loss, blood transfusion rate, operative time, length of hospital stay, and 30-day overall complication rate.

Results We identified 99 relevant articles (108 studies, 14,448 patients). For robotic versus OS, 50 studies (11 RCTs, 39 PROs) demonstrated reduction in blood loss [ratio of means (RoM) 0.505, $95 \%$ confidence interval (CI) 0.408-0.602], transfusion rate [risk ratio (RR) $0.272,95 \%$ CI 0.165-0.449], length of hospital stay (RoM 0.695, 0.615-0.774), and 30-day overall complication rate (RR $0.637,0.483-0.838)$ in favour of robotic surgery. For robotic versus MIS, 58 studies (21 RCTs, 37 PROs) demonstrated reduced blood loss (RoM 0.853, $0.736-0.969)$ and transfusion rate (RR 0.621, 0.390-0.988) in favour of robotic surgery but similar length of hospital stay
\end{abstract}

Hutan Ashrafian

h.ashrafian@imperial.ac.uk

1 Department of Surgery and Cancer, Imperial College London, 10th Floor QEQM Building, St. Mary's Hospital, London W2 1NY, UK

2 Institute of Global Health Innovation, Imperial College London, London SW7 2NA, UK
(RoM 0.982, 0.936-1.027) and 30-day overall complication rate (RR 0.988, 0.822-1.188). In both comparisons, robotic surgery prolonged operative time (OS: RoM 1.073, 1.022-1.124; MIS: RoM 1.135, 1.096-1.173). The benefits of robotic surgery lacked robustness on RCT-sensitivity analyses. However, many studies, including the relatively few available RCTs, suffered from high risk of bias and inadequate statistical power.

Conclusions Our results showed that robotic surgery contributed positively to some perioperative outcomes but longer operative times remained a shortcoming. Better quality evidence is needed to guide surgical decision making regarding the precise clinical targets of this innovation in the next generation of its use.

Keywords Robotic surgery - Conventional surgery · Perioperative outcomes

Robotic surgery represents a fundamental innovation in health care that is designed to enhance the quality of care for patients. Puma 560 was the first surgical robot applied in a clinical setting to obtain neurosurgical biopsies in 1985 [1]. The authors concluded that the robot contributed to improved accuracy. Since then, increasingly advanced surgical robots have been developed to assist in a rapidly expanding range of operative procedures and anatomical targets (Fig. 1). The drivers for continuous innovation stem from the potential to offer greater operative precision that may translate into enhanced clinical outcomes and the accompanying background of corporate revenues within the healthcare technology sector.

To achieve these goals, current robotic platforms are designed to incorporate advanced features, such as, (i) dexterous capability with accompanying instrumentation, (ii) 
Fig. 1 Timeline demonstrating selected events in the history and development of surgical robots

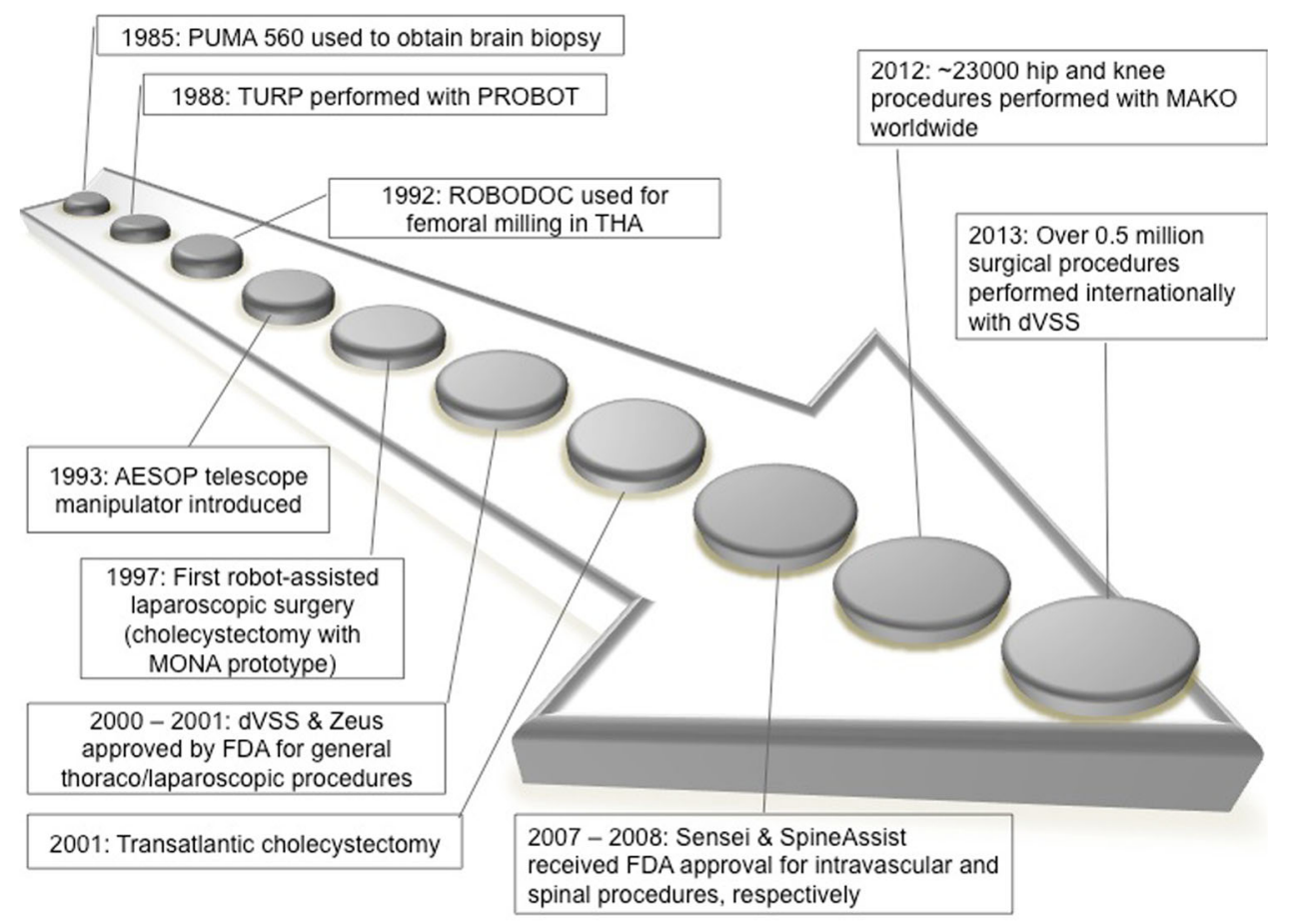

augmented visualisation, (iii) improved stability, (iv) natural coordination, (v) accurate cutting capacity, (vi) reliable execution, and (vii) enhanced surgeon ergonomics. These features can theoretically increase surgical precision by rendering difficult operative tasks easier to perform safely. Moreover, surgical robots have retained the capacity to enable surgery through smaller incisions. Collectively, these characteristics aim to enhance outcomes beyond that achievable through conventional operative methods.

The adoption and diffusion of robotic surgery demonstrate a positive trend in some geographical areas, particularly for advanced economies. This can be illustrated by the prominent application of the da Vinci ${ }^{\circledR}$ Surgical System (dVSS; Intuitive Surgical Inc., Mountain View, Sunnyvale, California, USA), which has US Food and Drug Administration (FDA) clearance across a multitude of specialties [2], demonstrating its greatest exposure for urological and gynaecological procedures [3]. For example, more than half of radical prostatectomies and about a third of benign hysterectomies are already performed robotically in the USA $[3,4]$.

Despite offering some elements of innovative technology, the necessary evidence to justify the expanding investment in robotic surgery remains ambiguous. Whilst the concept of robotic surgery is almost universally favoured, its widespread promotion across all healthcare sectors requires robust justification, not least because it can be very costly [5]. Studies comparing outcomes of robotic surgery with conventional approaches for specific robots and procedures are certainly not scarce. However, the systematic assessment of robotic surgery collectively as a single entity has not been performed. As we approach the end of the third decade following the pioneering use of the first surgical robot, an overview of this innovation may be useful for understanding the adoption of innovations in health care.

The aim of this comprehensive systematic review and meta-analysis was to draw evidence from comparative studies in robotic surgery, regardless of specialty and procedure type, and irrespective of patient age and sex. We avoided the biases of retrospective studies that dominate the literature by focussing only on randomised controlled trials (RCTs) and non-randomised prospective studies. In comparing potentially very heterogeneous studies, we emphasised a methodology that identified the proportional benefit of robotic surgical outcomes compared with controls in each study. This offered internal consistency from each study. We were then able to calculate a pooled proportional benefit for specific robotic surgical outcomes for all studies.

In this review, we evaluated core perioperative variables as our primary outcomes. These were (i) blood loss, (ii) blood transfusion rate, (iii) operative time, (iv) length of hospital stay, and (v) 30-day overall complication rate. In robotic surgical studies, these perioperative variables were most commonly addressed. Analyses were performed separately for robotic versus open surgery (OS) and robotic versus minimally invasive surgery (MIS). As a secondary outcome, we calculated the proportion of studies that demonstrated adequate statistical power for the evaluation of these clinical outcomes. 


\section{Materials and methods}

This review was performed according to the Preferred Reporting Items for Systematic Reviews and Meta-analyses (PRISMA) statement [6].

\section{Inclusion and exclusion criteria}

We defined surgery as any interventional procedure involving alteration in anatomy and that either requires a skin (or mucosal) incision or puncture. Patients requiring surgery for which a robotic approach was a feasible alternative approach to OS or MIS were included. There was no age or sex restriction. Controls were eligible only if patients underwent surgery and no robotic assistance was provided. RCTs and prospective studies that addressed one or more core perioperative surgical outcomes (blood loss, blood transfusion rate, operative time, length of hospital stay, and 30-day overall complication rate) were included. For operative time, we included studies that explicitly defined it as starting from skin incision to skin closure (for intravascular procedures, we used procedure time, which was generally defined as time from first venous puncture to sheath withdrawal at the end of the procedure). Whilst this measure does not represent the total theatre occupation time, it was selected to improve comparability because operative time was variedly defined in the literature.

We excluded studies where surgical robots were used for stereotactic, endoscopic, or single-incision laparoscopic surgery. Robotic instrument positioners without concurrent use of other robotic instrumentation tools were also excluded, as were innovations that are generally not considered robotic technology, such as remote magnetic catheter navigation and pure computer navigation systems. We also discounted studies with historical controls that preceded the robotic arm considerably (that is, greater than a year) as well as those that retrospectively reviewed and analysed prospective databases. Laboratory studies involving synthetic models, animals, or cadavers were not considered.

\section{Search methodology}

Using the OvidSP search engine, the MEDLINE, EMBASE, and PsycINFO databases were searched on 2 September 2013 with the terms: robot* (tw) AND [intervention* (tw) OR surg* (tw)]. The same search terms were used to search the ClinicalTrials.gov registry to identify potentially relevant trials. On 26 May 2014, these trials were reviewed to identify any relevant published data. To avoid losing generally older papers which had used the term computer-assisted instead of robot, we also performed a search on 7 October 2013 with the terms: [surgery, computer-assisted
(MESH, exp) OR computer-assisted surg* (tw) OR computer-aided surg* (tw)] AND [intervention* (tw) OR surg* (tw)]. Studies from 1990 to the search dates were included. There was no language restriction. Relevant review articles, including health technology assessments, found through our search strategy were also hand-searched to identify any remaining studies.

\section{Data collection and analysis}

\section{Study selection}

Articles were screened from titles and abstracts by three authors independently (AT, SM, and AS). Potentially relevant articles that appear to fit the inclusion and exclusion criteria were obtained in full text. These were independently assessed for eligibility by the same authors. Articles were excluded if they had duplicate or incomplete data, or if they were only available in abstract form. Any disagreement was resolved through discussion with a senior author (HA).

\section{Dealing with duplicate publications}

If several articles reported outcomes from a single study, the article with the most comprehensive results (most number of patients and/or most recent publication) was included. If this article failed to report outcomes that were otherwise available in the duplicate article, then the additional data from the duplicate article were included.

\section{Data extraction}

One author (AT) extracted data into an Excel 2011 database (Microsoft Corp., Redmond, Washington, USA), which were then reviewed independently by three authors (SM, AS, and HA). For each article, the year of publication, study design, total number of patients, number of patients in each arm, robot and control type, baseline characteristics, and results of outcome measures of interest were extracted. For continuous outcomes, we extracted the mean and standard deviation (or if unavailable, the median and standard error, range, or interquartile range). For categorical outcomes, we recorded the number of events.

\section{Risk of bias assessment}

Three authors (AT, SM, and AS) independently assessed the risk of bias of eligible articles. Quality of articles with more than one study was assessed on their overall methodology. The Cochrane risk of bias tool [7] was applied to RCTs. Seven key domains were assessed: method of random sequence generation, allocation concealment, blinding of 
participants and personnel, blinding of outcome assessors, completeness of outcome data, selective reporting, and other potential sources of bias. Based on a set of listed criteria, each domain was judged to have either a low, high, or unclear risk of bias. If a study had unclear or high risk of bias for one or more key domains, then it was classified as having, respectively, an unclear or high risk of bias overall. If instead all the key domains had low bias risk, then the study was judged to have a low risk of bias overall [7].

For prospective studies, the Newcastle-Ottawa scale (NOS) [8] was used for quality scoring. The NOS judges studies on three categories: the selection of the study groups (comprising four numbered items: representativeness of exposed cohort, selection of non-exposed cohort, ascertainment of exposure, demonstration that outcomes were not present at start of study), the comparability of the groups (comprising one numbered item: comparability of cohorts on basis of study design or analysis), and outcomes (comprising three numbered items: assessment of outcome, appropriateness of length of follow-up, adequacy of follow-up of cohorts). From a set of listed criteria, a maximum of one star can be awarded for each numbered item, except for comparability where a maximum of two stars can be awarded. The possible NOS score ranges from 0 to 9 stars. We classified studies with $\geq 7$ stars as "higher" quality and $<7$ stars as "lower" quality.

Risk of bias assessment was made at the level of outcomes. We assessed perioperative outcomes together as a class [7, 9]. If a study addressed several perioperative outcomes, the risk of bias for a particular domain was judged based on the outcome that was most affected by the study methodology. Any disagreement with risk of bias assessment was resolved through discussion with a senior author (HA).

\section{Data synthesis and statistical methods}

Meta-analysis was based on control type, that is, either robotic versus OS or robotic versus MIS. Wherever possible, we used results from intention-to-treat analyses. Continuous outcomes were analysed by calculating the ratio of means (RoM) for each study, with expression of uncertainty of each result represented by the $95 \%$ confidence intervals (CI) [10]. We substituted median for mean in studies where only the median was reported. When the calculated RoM was 1 , computation was not possible. Consequently, these results were excluded. Categorical outcomes were analysed using risk ratio (RR) with $95 \%$ CI [7]. Studies reporting categorical outcomes with no events in both the robotic and control groups were excluded, as their effect sizes were not computable. We performed meta-analysis if two or more separate studies were available. The inverse-variance, random-effects model of DerSimonian and Laird [11] was used for both continuous and categorical outcomes. This was accomplished using Stata 13 (StataCorp., College Station, Texas, USA). Sensitivity analysis on RCTs was also performed. The $I^{2}$ statistic was used to estimate the degree of heterogeneity between studies, where larger values indicate increasing heterogeneity [12].

Post hoc power analysis (significant at the $5 \%$ level, two-tailed $\mathrm{t}$ test) was conducted for all eligible studies using the $G^{*}$ Power 3.1 programme [13]. Power was calculated for large $(d=0.8)$, medium $(d=0.5)$, and small $(d=0.2)$ effect sizes. We defined adequate statistical power as $>80 \%$. We also identified studies with clearly specified primary outcomes and where power analysis was performed to determine the required sample size for adequate assessment of these outcomes.

\section{Results}

\section{Search results}

A total of 43,132 articles were identified from the databases. This included 104 trials from the ClinicalTrials.gov registry, of which one [14] was subsequently found to contain relevant published data. After removing duplicates, 28,574 articles were screened based on their titles and abstracts. Of these, 1702 potentially relevant full-text articles were retrieved for further evaluation. We found 97 articles that met the inclusion criteria. Two additional articles were identified through hand-searching. In total, 99 articles, involving 14,448 patients overall, were included in this review (Fig. 2).

\section{Description of included studies}

Of the included articles, all but one [15] investigated outcomes in adult patients. Overall, there were 31 and 68 articles, respectively, that were based on RCT and nonrandomised prospective comparative designs. They encompassed a wide range of specialties and procedures (Tables 1, 2). Some articles comprised more than one comparison or study [16-23].

\section{Robotic versus OS}

For robotic versus OS, there were 50 studies (11 RCTs and 39 prospective studies) (Table 1). The year of publication ranged from 1998 to 2013. In total, there were 5910 and 4237 patients in the robotic and OS groups, respectively. The smallest and largest sample sizes were 14 and 1738, respectively. The surgical robots used in these studies were the dVSS, Zeus ${ }^{\circledR}$ Robotic Surgical System (ZRSS; Computer Motion Inc., Santa Barbara, California, USA), ROBODOC ${ }^{\circledR}$ Surgical System (Curexo Technology Corp., Fremont, 
Fig. 2 Flow chart of included studies. *Some articles contained more than one comparison or study (see text). $O S$ open surgery, MIS minimally invasive surgery, $R C T$ randomised controlled trial

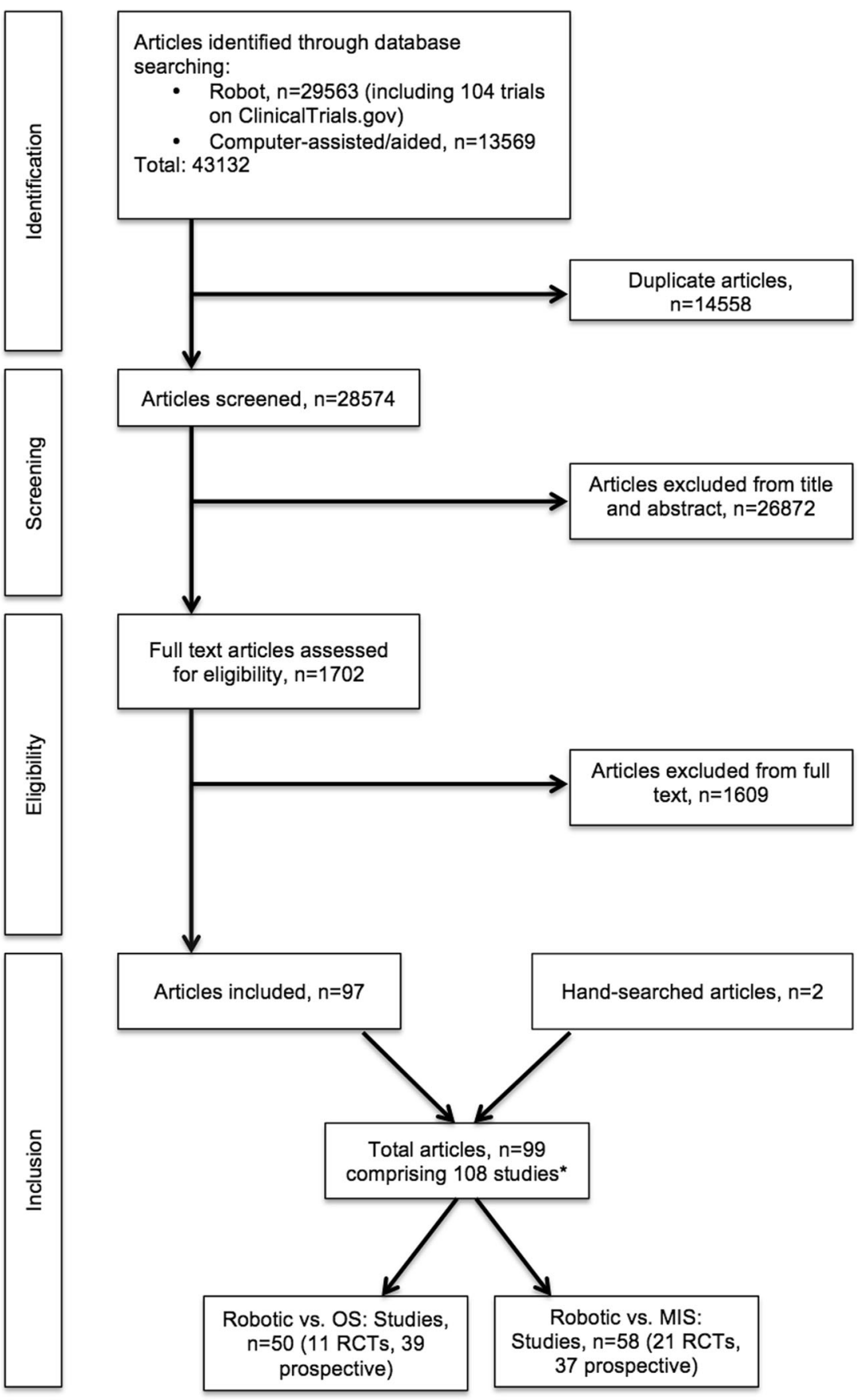

California, USA), Acrobot ${ }^{\circledR}$ Surgical System (The Acrobot Co. Ltd., London, UK), CASPAR system (OrtoMaquet, Rastatt, Germany), and SpineAssist ${ }^{\circledR}$ (Mazor Robotics Ltd., Caesarea, Israel).

\section{Robotic versus MIS}

For robotic versus MIS, there were 58 studies (21 RCTs and 37 prospective studies), which were published between 2001 and 2014 (Table 2). Taking into account all studies, the robotic and MIS groups consisted of 1991 and 2310 patients, respectively. Sample sizes ranged from 12 to 390 . The surgical robots used were the dVSS, ZRSS, Mona (Intuitive Surgical), and Sense $i^{\circledR}$ Robotic Catheter System (Hansen Medical Inc., Mountain View, California, USA).

\section{Risk of bias assessment}

All included articles were assessed for the quality of their methodology. Of note, all 31 RCT articles suffered from a high risk of bias because they all showed a high risk of bias in the performance bias domain (Fig. 3). This was primarily 
Table 1 Studies comparing robotic versus open surgery

\begin{tabular}{|c|c|c|c|c|c|c|c|c|c|c|}
\hline \multirow[t]{3}{*}{ References } & \multirow[t]{3}{*}{ Procedure } & \multirow[t]{3}{*}{ Design } & \multicolumn{3}{|c|}{ No. of patients, $n$} & \multirow[t]{3}{*}{ Robot } & \multirow{3}{*}{$\begin{array}{l}\text { Perioperative } \\
\text { outcomes addressed }^{\mathrm{a}}\end{array}$} & \multirow{2}{*}{\multicolumn{3}{|c|}{$\frac{\text { Power }}{\text { Effect size }}$}} \\
\hline & & & \multirow[t]{2}{*}{ Total } & \multirow[t]{2}{*}{$\mathrm{R}$} & \multirow[t]{2}{*}{$\mathrm{C}$} & & & & & \\
\hline & & & & & & & & Large & Medium & Small \\
\hline $\begin{array}{l}\text { Bertani et al. } \\
{[16]^{\mathrm{b}}}\end{array}$ & Rectal resection & PRO & 86 & 52 & 34 & dVSS & BL, LOS, C & 0.948 & 0.611 & 0.146 \\
\hline Kim et al. [68] & Rectal resection & PRO & 200 & 100 & 100 & dVSS & BT, LOS, C & 1.000 & 0.940 & 0.291 \\
\hline $\begin{array}{l}\text { Bertani et al. } \\
{[16]^{\mathrm{b}}}\end{array}$ & Colectomy & PRO & 79 & 34 & 45 & dVSS & BL, LOS, C & 0.935 & 0.584 & 0.140 \\
\hline Lee et al. [47] & Thyroidectomy & PRO & 84 & 41 & 43 & dVSS & BL, OT, LOS, C & 0.952 & 0.619 & 0.148 \\
\hline Kim et al. [48] & Thyroidectomy & PRO & 37 & 19 & 18 & dVSS & BL & 0.657 & 0.315 & 0.091 \\
\hline Ryu et al. [96] & Thyroidectomy & PRO & 90 & 45 & 45 & dVSS & LOS, C & 0.964 & 0.738 & 0.180 \\
\hline Menon et al. [34] & Prostatectomy & PRO & 60 & 30 & 30 & dVSS & BL, BT, OT, LOS, C & 0.861 & 0.478 & 0.119 \\
\hline Tewari et al. [36] & Prostatectomy & PRO & 300 & 200 & 100 & dVSS & BL, BT, OT, LOS, C & 1.000 & 0.983 & 0.370 \\
\hline $\begin{array}{l}\text { Farnham et al. } \\
\text { [31] }\end{array}$ & Prostatectomy & PRO & 279 & 176 & 103 & $\mathrm{dVSS}$ & $\mathrm{BL}, \mathrm{BT}$ & 1.000 & 0.980 & 0.362 \\
\hline Wood et al. [35] & Prostatectomy & PRO & 206 & 117 & 89 & dVSS & BL, BT, LOS, C & 1.000 & 0.943 & 0.293 \\
\hline Nelson et al. [95] & Prostatectomy & $\mathrm{PRO}$ & 1003 & 629 & 374 & dVSS & LOS, C & 1.000 & 1.000 & 0.864 \\
\hline Ham et al. [37] & Prostatectomy & PRO & 298 & 188 & 110 & dVSS & BL, LOS, C & 1.000 & 0.986 & 0.383 \\
\hline Ficarra et al. [32] & Prostatectomy & PRO & 208 & 103 & 105 & dVSS & BL, BT, LOS, C & 1.000 & 0.948 & 0.300 \\
\hline $\begin{array}{l}\text { Carlsson et al. } \\
\text { [111] }\end{array}$ & Prostatectomy & PRO & 1738 & 1253 & 485 & dVSS & $\mathrm{C}$ & 1.000 & 1.000 & 0.962 \\
\hline Hong et al. [38] & Prostatectomy & PRO & 51 & 26 & 25 & dVSS & $\mathrm{BL}, \mathrm{BT}$ & 0.799 & 0.417 & 0.108 \\
\hline $\begin{array}{l}\text { Doumerc et al. } \\
\text { [69] }\end{array}$ & Prostatectomy & PRO & 714 & 212 & 502 & dVSS & BT, LOS, C & 1.000 & 1.000 & 0.684 \\
\hline Kordan et al. [33] & Prostatectomy & PRO & 1244 & 830 & 414 & dVSS & $\mathrm{BL}, \mathrm{BT}$ & 1.000 & 1.000 & 0.913 \\
\hline $\begin{array}{l}\text { Di Pierro et al. } \\
\text { [70] }\end{array}$ & Prostatectomy & PRO & 150 & 75 & 75 & dVSS & $\mathrm{BT}, \mathrm{C}$ & 0.998 & 0.860 & 0.229 \\
\hline Kim et al. [112] & Prostatectomy & PRO & 763 & 528 & 235 & dVSS & $\mathrm{C}$ & 1.000 & 1.000 & 0.721 \\
\hline $\begin{array}{l}\text { Ludovico et al. } \\
\text { [39] }\end{array}$ & Prostatectomy & PRO & 130 & 82 & 48 & dVSS & BL, LOS, C & 0.992 & 0.780 & 0.194 \\
\hline Rhee et al. [42] & Cystectomy & PRO & 30 & 7 & 23 & dVSS & BL, BT, OT, LOS & 0.432 & 0.201 & 0.073 \\
\hline Nix et al. [25] & Cystectomy & RCT & 41 & 21 & 20 & dVSS & $\mathrm{BL}, \mathrm{LOS}, \mathrm{C}$ & 0.704 & 0.345 & 0.096 \\
\hline $\mathrm{Ng}$ et al. [41] & Cystectomy & PRO & 187 & 83 & 104 & dVSS & $\mathrm{BL}, \mathrm{OT}, \mathrm{LOS}, \mathrm{C}^{\mathrm{c}}$ & 1.000 & 0.922 & 0.272 \\
\hline Martin et al. [40] & Cystectomy & PRO & 33 & 19 & 14 & dVSS & BL, LOS & 0.595 & 0.280 & 0.085 \\
\hline Khan et al. [17] ${ }^{\mathrm{b}}$ & Cystectomy & PRO & 100 & 48 & 52 & dVSS & BL, BT, OT, LOS, C & 0.977 & 0.696 & 0.167 \\
\hline Parekh et al. [26] & Cystectomy & $\mathrm{RCT}$ & 40 & 20 & 20 & dVSS & BL, BT, OT, LOS, C & 0.693 & 0.338 & 0.095 \\
\hline $\begin{array}{l}\text { Masson-Lecomte } \\
\text { et al. [49] }\end{array}$ & Nephrectomy & PRO & 100 & 42 & 58 & dVSS & BL, BT, OT, LOS, C & 0.974 & 0.686 & 0.165 \\
\hline $\begin{array}{l}\text { Parekattil et al. } \\
{[23]^{\mathrm{b}}}\end{array}$ & Vasovasostomy & PRO & 94 & 66 & 28 & dVSS & OT & 0.939 & 0.592 & 0.142 \\
\hline $\begin{array}{l}\text { Parekattil et al. } \\
{[23]^{\mathrm{b}}}\end{array}$ & Vasoepididymostomy & PRO & 61 & 44 & 17 & dVSS & OT & 0.787 & 0.406 & 0.106 \\
\hline $\begin{array}{l}\text { Bucerius et al. } \\
{[18]^{\mathrm{b}}}\end{array}$ & CABG & PRO & 117 & 24 & 93 & dVSS & LOS & 0.934 & 0.581 & 0.139 \\
\hline Kiaii et al. [94] & CABG & PRO & 100 & 50 & 50 & ZRSS & LOS, C & 0.977 & 0.697 & 0.168 \\
\hline Poston et al. [43] & CABG & PRO & 200 & 100 & 100 & dVSS & BL, LOS, C & 1.000 & 0.940 & 0.291 \\
\hline $\begin{array}{l}\text { Bachinsky et al. } \\
{[71]}\end{array}$ & CABG & PRO & 52 & 25 & 27 & dVSS & BT, LOS & 0.807 & 0.424 & 0.109 \\
\hline $\begin{array}{l}\text { Balduyck et al. } \\
\text { [110] }\end{array}$ & $\begin{array}{l}\text { Anterior mediastinal } \\
\text { mass resection }\end{array}$ & PRO & 36 & 14 & 22 & dVSS & $\mathrm{C}$ & 0.623 & 0.295 & 0.088 \\
\hline $\begin{array}{l}\text { Hoekstra et al. } \\
{[20]^{\mathrm{b}}}\end{array}$ & $\begin{array}{l}\text { Endometrial cancer } \\
\text { staging }\end{array}$ & PRO & 58 & 32 & 26 & dVSS & BL, OT, LOS, C & 0.846 & 0.461 & 0.116 \\
\hline
\end{tabular}


Table 1 continued

\begin{tabular}{|c|c|c|c|c|c|c|c|c|c|c|}
\hline \multirow[t]{3}{*}{ References } & \multirow[t]{3}{*}{ Procedure } & \multirow[t]{3}{*}{ Design } & \multicolumn{3}{|c|}{ No. of patients, $n$} & \multirow[t]{3}{*}{ Robot } & \multirow{3}{*}{$\begin{array}{l}\text { Perioperative } \\
\text { outcomes addressed }^{\text {a }}\end{array}$} & \multirow{2}{*}{\multicolumn{3}{|c|}{$\frac{\text { Power }}{\text { Effect size }}$}} \\
\hline & & & \multirow[t]{2}{*}{ Total } & \multirow[t]{2}{*}{$\mathrm{R}$} & \multirow[t]{2}{*}{$\mathrm{C}$} & & & & & \\
\hline & & & & & & & & Large & Medium & Small \\
\hline $\begin{array}{l}\text { Göçmen et al. } \\
\text { [45] }\end{array}$ & $\begin{array}{l}\text { Endometrial cancer } \\
\text { staging }\end{array}$ & PRO & 22 & 10 & 12 & dVSS & BL, BT, OT, LOS, C & 0.428 & 0.199 & 0.073 \\
\hline Jung et al. $[19]^{\mathrm{b}}$ & $\begin{array}{l}\text { Endometrial cancer } \\
\text { staging }\end{array}$ & $\mathrm{PRO}$ & 84 & 28 & 56 & dVSS & BT, OT, LOS, C & 0.927 & 0.569 & 0.137 \\
\hline Lowe et al. [46] & $\begin{array}{l}\text { Hysterectomy- } \\
\text { cervical cancer }\end{array}$ & PRO & 14 & 7 & 7 & dVSS & BL, OT, LOS, C & 0.281 & 0.139 & 0.064 \\
\hline Collins et al. [44] & Sacrocolpopexy & PRO & 48 & 30 & 18 & dVSS & $\mathrm{BL}$ & 0.748 & 0.375 & 0.101 \\
\hline Bargar et al. [28] & Total hip arthroplasty & RCT & 136 & 70 & 66 & ROBODOC & BL, LOS, C & 0.996 & 0.825 & 0.212 \\
\hline Bach et al. [109] & Total hip arthroplasty & PRO & 50 & 25 & 25 & ROBODOC & $\mathrm{C}$ & 0.791 & 0.410 & 0.107 \\
\hline Honl et al. [76] & Total hip arthroplasty & $\mathrm{RCT}$ & 141 & 61 & 80 & ROBODOC & OT, C & 0.997 & 0.832 & 0.215 \\
\hline Siebel et al. [113] & Total hip arthroplasty & PRO & 71 & 36 & 35 & CASPAR & $\mathrm{C}$ & 0.914 & 0.547 & 0.132 \\
\hline $\begin{array}{l}\text { Nishihara et al. } \\
\text { [27] }\end{array}$ & Total hip arthroplasty & $\mathrm{RCT}$ & 156 & 78 & 78 & ROBODOC & $\mathrm{BL}, \mathrm{BT}$ & 0.999 & 0.873 & 0.237 \\
\hline $\begin{array}{l}\text { Nakamura et al. } \\
\text { [107] }\end{array}$ & Total hip arthroplasty & $\mathrm{RCT}$ & 146 & 75 & 71 & ROBODOC & $\mathrm{C}$ & 0.998 & 0.851 & 0.224 \\
\hline Cobb et al. [106] & $\begin{array}{l}\text { Unicompartmental } \\
\text { knee arthroplasty }\end{array}$ & $\mathrm{RCT}$ & 28 & 13 & 15 & Acrobot & $\mathrm{C}$ & 0.529 & 0.246 & 0.080 \\
\hline Park et al. [108] & Total knee arthroplasty & $\mathrm{RCT}$ & 62 & 32 & 30 & ROBODOC & $\mathrm{C}$ & 0.872 & 0.490 & 0.121 \\
\hline Song et al. [29] & Total knee arthroplasty & RCT & 60 & 30 & 30 & ROBODOC & $\mathrm{BL}, \mathrm{C}$ & 0.861 & 0.478 & 0.119 \\
\hline Song et al. [30] & Total knee arthroplasty & RCT & 100 & 50 & 50 & ROBODOC & $\mathrm{BL}, \mathrm{C}$ & 0.977 & 0.697 & 0.168 \\
\hline Ringel et al. [77] & $\begin{array}{l}\text { Spinal pedicle screw } \\
\text { insertion }\end{array}$ & RCT & 60 & 30 & 30 & SpineAssist & OT, LOS & 0.861 & 0.478 & 0.119 \\
\hline Total & & & 10,147 & 5910 & 4237 & & & & & \\
\hline
\end{tabular}

$B L$ blood loss, $B T$ blood transfusion rate, $O T$ skin-to-skin operative (or procedure) time, $L O S$ length of hospital stay, $C$ 30-day overall complication rate, $C A B G$ coronary artery bypass grafting, $R C T$ randomised controlled trial, $P R O$ non-randomised prospective comparative studies

${ }^{a}$ Relevant to this review

${ }^{b}$ More than one comparison or study in an article

c Not computable, as there were more complications than the number of patients in the open group-complication data were excluded from meta-analysis as a result; for robotic studies on hips and knees, $n=$ number of limbs

due to the lack of surgeon blinding, which is unlikely to be possible in clinical trials of robotic surgery. As perioperative outcomes are especially vulnerable to performance bias, this risk was judged to be high. The subject of patient blinding, which is difficult in surgical trials but potentially feasible [24], was frequently unaddressed or unreported by authors. Most RCTs showed low risk of attrition bias, with complete perioperative outcome data. In many trials, however, the risk of bias related to sequence generation, allocation concealment, blinding of outcome assessor, and selective reporting was unclear, as sufficient information was not available due to poor reporting.

Of 68 articles of non-randomised prospective design, 55 $(80.9 \%)$ were of "higher" quality (Tables 3, 4). All prospective studies met the criteria for ascertainment of exposure, absence of outcome at the start of study, outcome assessment, and duration of follow-up. Most prospective studies also selected their control cohort from the same community as the robotic cohort and showed adequate follow-up. Many of them suffered from poor comparability, as expected from the lack of randomisation where selection bias is a caveat. In some cases, the representativeness of the robotic cohort in the community was felt not be adequate.

\section{Meta-analyses of perioperative surgical outcomes}

\section{(i) Blood loss}

\section{Robotic versus $O S$}

There were six RCT [25-30] and 23 prospective [16, 17, 20, 31-49] studies reporting on blood loss, giving a total of 29 studies overall. Meta-analysis demonstrated blood loss 
Table 2 Studies comparing robotic versus minimally invasive surgery

\begin{tabular}{|c|c|c|c|c|c|c|c|c|c|c|}
\hline \multirow[t]{3}{*}{ References } & \multirow[t]{3}{*}{ Procedure } & \multirow[t]{3}{*}{ Design } & \multicolumn{3}{|c|}{ No. of patients, $n$} & \multirow[t]{3}{*}{ Robot } & \multirow{3}{*}{$\begin{array}{l}\text { Perioperative } \\
\text { outcomes addressed }^{\mathrm{a}}\end{array}$} & \multirow{2}{*}{\multicolumn{3}{|c|}{$\frac{\text { Power }}{\text { Effect size }}$}} \\
\hline & & & \multirow[t]{2}{*}{ Total } & \multirow[t]{2}{*}{$\mathrm{R}$} & \multirow[t]{2}{*}{$\mathrm{C}$} & & & & & \\
\hline & & & & & & & & Large & Medium & Small \\
\hline Pigazzi et al. [56] & Rectal resection & PRO & 12 & 6 & 6 & $\mathrm{dVSS}$ & BL, LOS, C & 0.241 & 0.123 & 0.061 \\
\hline Patriti et al. [55] & Rectal resection & PRO & 66 & 29 & 37 & dVSS & BL, BT, LOS, C & 0.888 & 0.510 & 0.125 \\
\hline Baik et al. [89] & Rectal resection & PRO & 113 & 56 & 57 & $\mathrm{dVSS}$ & OT, LOS, C & 0.984 & 0.726 & 0.176 \\
\hline Kim et al. [75] & Rectal resection & PRO & 209 & 62 & 147 & dVSS & BT, OT, LOS, C & 1.000 & 0.908 & 0.260 \\
\hline Bertani et al. $[16]^{b}$ & Colectomy & PRO & 64 & 34 & 30 & dVSS & $\mathrm{BL}, \mathrm{LOS}, \mathrm{C}$ & 0.882 & 0.502 & 0.123 \\
\hline Park et al. [51] & Colectomy & $\mathrm{RCT}$ & 70 & 35 & 35 & dVSS & BL, BT, LOS, C & 0.910 & 0.541 & 0.131 \\
\hline Jiménez Rodríguez et al. [74] & $\begin{array}{r}\text { Colorectal } \\
\text { resection }\end{array}$ & RCT & 56 & 28 & 28 & dVSS & BT, LOS, C & 0.836 & 0.451 & 0.114 \\
\hline Heemskerk et al. [102] & Rectopexy & PRO & 33 & 14 & 19 & dVSS & LOS & 0.595 & 0.280 & 0.085 \\
\hline Wong et al. [57] & Rectopexy & PRO & 63 & 23 & 40 & dVSS & BL, LOS, C & 0.853 & 0.468 & 0.117 \\
\hline Cadière et al. [97] & Fundoplication & RCT & 21 & 10 & 11 & Mona & LOS, C & 0.412 & 0.193 & 0.072 \\
\hline Melvin et al. [84] & Fundoplication & PRO & 40 & 20 & 20 & dVSS & OT, $\mathrm{C}$ & 0.693 & 0.338 & 0.095 \\
\hline Draaisma et al. [50] & Fundoplication & $\mathrm{RCT}$ & 50 & 25 & 25 & dVSS & BL, OT, LOS, C & 0.791 & 0.410 & 0.107 \\
\hline Morino et al. [78] & Fundoplication & $\mathrm{RCT}$ & 50 & 25 & 25 & dVSS & OT, LOS, C & 0.791 & 0.410 & 0.107 \\
\hline Nakadi et al. [99] & Fundoplication & $\mathrm{RCT}$ & 20 & 9 & 11 & dVSS & LOS, C & 0.392 & 0.184 & 0.071 \\
\hline Lehnert et al. [15] & Fundoplication & PRO & 20 & 10 & 10 & dVSS & OT, C & 0.395 & 0.185 & 0.071 \\
\hline Müller-Stitch et al. [98] & Fundoplication & RCT & 40 & 20 & 20 & dVSS & LOS, C & 0.693 & 0.338 & 0.095 \\
\hline Hartmann et al. [101] & Fundoplication & PRO & 80 & 18 & 62 & dVSS & LOS, C & 0.839 & 0.454 & 0.114 \\
\hline Sanchez et al. [100] & RYGB & $\mathrm{RCT}$ & 50 & 25 & 25 & dVSS & LOS, C & 0.791 & 0.410 & 0.107 \\
\hline Benizri et al. [85] & RYGB & PRO & 200 & 100 & 100 & dVSS & OT, LOS, C & 1.000 & 0.940 & 0.291 \\
\hline Mühlmann et al. [104] & Various bariatric $^{c}$ & PRO & 20 & 10 & 10 & dVSS & LOS, C & 0.395 & 0.185 & 0.071 \\
\hline Park et al. [91] & Gastrectomy & PRO & 150 & 30 & 120 & dVSS & OT, LOS, C & 0.973 & 0.682 & 0.164 \\
\hline Ruurda et al. [79] & Cholecystectomy & $\mathrm{RCT}$ & 20 & 10 & 10 & dVSS & OT & 0.395 & 0.185 & 0.071 \\
\hline Nio et al. [105] & Cholecystectomy & PRO & 20 & 10 & 10 & ZRSS & LOS, C & 0.395 & 0.185 & 0.071 \\
\hline Zhou et al. [52] & Cholecystectomy & $\mathrm{RCT}$ & 40 & 20 & 20 & ZRSS & BL, LOS, C & 0.693 & 0.338 & 0.095 \\
\hline Kornprat et al. [90] & Cholecystectomy & PRO & 46 & 20 & 26 & ZRSS & OT & 0.749 & 0.376 & 0.101 \\
\hline Berber et al. [58] & Liver resection & PRO & 32 & 9 & 23 & dVSS & $\mathrm{BL}, \mathrm{OT}, \mathrm{C}$ & 0.504 & 0.234 & 0.078 \\
\hline Brunaud et al. [93] & Adrenalectomy & PRO & 28 & 14 & 14 & dVSS & OT, LOS, C & 0.531 & 0.247 & 0.080 \\
\hline Morino et al. [81] & Adrenalectomy & RCT & 20 & 10 & 10 & dVSS & OT, LOS, C & 0.395 & 0.185 & 0.071 \\
\hline Wu et al. [66] & Adrenalectomy & PRO & 12 & 5 & 7 & ZRSS & $\mathrm{BL}, \mathrm{LOS}, \mathrm{C}$ & 0.236 & 0.121 & 0.061 \\
\hline Ploussard et al. [59] & Prostatectomy & PRO & 288 & 83 & 205 & $\mathrm{dVSS}$ & $\mathrm{BL}, \mathrm{BT}, \mathrm{LOS}, \mathrm{C}$ & 1.000 & 0.969 & 0.335 \\
\hline Gosseine et al. [67] & Prostatectomy & PRO & 247 & 122 & 125 & dVSS & $\mathrm{BL}, \mathrm{BT}, \mathrm{LOS}, \mathrm{C}$ & 1.000 & 0.975 & 0.347 \\
\hline Asimakopoulos et al. [72] & Prostatectomy & RCT & 112 & 52 & 60 & dVSS & BT, C & 0.987 & 0.744 & 0.182 \\
\hline Porpiglia et al. [53] & Prostatectomy & $\mathrm{RCT}$ & 120 & 60 & 60 & dVSS & BL, OT, LOS, C & 0.991 & 0.775 & 0.192 \\
\hline Khan et al. $[17]^{\mathrm{b}}$ & Cystectomy & PRO & 106 & 48 & 58 & dVSS & BL, BT, OT, LOS, C & 0.982 & 0.719 & 0.174 \\
\hline Caruso et al. [63] & Nephrectomy & PRO & 20 & 10 & 10 & dVSS & $\mathrm{BL}, \mathrm{BT}, \mathrm{LOS}, \mathrm{C}$ & 0.395 & 0.185 & 0.071 \\
\hline Hemal et al. [60] & Nephrectomy & PRO & 30 & 15 & 15 & dVSS & BL, BT, LOS, C & 0.562 & 0.262 & 0.083 \\
\hline Kural et al. [62] & Nephrectomy & PRO & 31 & 11 & 20 & $\mathrm{dVSS}$ & BL, BT, LOS, C & 0.540 & 0.251 & 0.081 \\
\hline Masson-Lecomte et al. [61] & Nephrectomy & PRO & 265 & 220 & 45 & $\mathrm{dVSS}$ & BL, BT, LOS, C & 0.998 & 0.861 & 0.230 \\
\hline Bucerius et al. [18] ${ }^{\mathrm{b}}$ & CABG & PRO & 97 & 24 & 73 & dVSS & LOS & 0.920 & 0.557 & 0.134 \\
\hline Mierdl et al. [92] & CABG & PRO & 46 & 30 & 16 & dVSS & OT, C & 0.715 & 0.352 & 0.097 \\
\hline Sarlos et al. [54] & $\begin{array}{r}\text { Hysterectomy- } \\
\text { benign disease }\end{array}$ & RCT & 95 & 47 & 48 & dVSS & BL, OT, LOS, C & 0.971 & 0.674 & 0.162 \\
\hline Paraiso et al. [73] & $\begin{array}{l}\text { Hysterectomy- } \\
\text { benign disease }\end{array}$ & $\mathrm{RCT}$ & 52 & 26 & 26 & dVSS & BT, OT & 0.807 & 0.424 & 0.109 \\
\hline Hoekstra et al. [20] ${ }^{\mathrm{b}}$ & $\begin{array}{l}\text { Endometrial cancer } \\
\text { staging }\end{array}$ & PRO & 39 & 32 & 7 & dVSS & BL, OT, LOS, C & 0.463 & 0.215 & 0.075 \\
\hline
\end{tabular}


Table 2 continued

\begin{tabular}{|c|c|c|c|c|c|c|c|c|c|c|}
\hline \multirow[t]{3}{*}{ References } & \multirow[t]{3}{*}{ Procedure } & \multirow[t]{3}{*}{ Design } & \multicolumn{3}{|c|}{ No. of patients, $n$} & \multirow[t]{3}{*}{ Robot } & \multirow{3}{*}{$\begin{array}{l}\text { Perioperative } \\
\text { outcomes addressed }^{\text {a }}\end{array}$} & \multirow{2}{*}{\multicolumn{3}{|c|}{$\frac{\text { Power }}{\text { Effect size }}$}} \\
\hline & & & \multirow[t]{2}{*}{ Total } & \multirow[t]{2}{*}{$\mathrm{R}$} & \multirow[t]{2}{*}{$\mathrm{C}$} & & & & & \\
\hline & & & & & & & & Large & Medium & Small \\
\hline Jung et al. $[19]^{\mathrm{b}}$ & $\begin{array}{l}\text { Endometrial cancer } \\
\text { staging }\end{array}$ & PRO & 53 & 28 & 25 & dVSS & BT, OT, LOS, C & 0.814 & 0.430 & 0.110 \\
\hline Paraiso et al. [80] & Sacrocolpopexy & $\mathrm{RCT}$ & 68 & 35 & 33 & dVSS & OT, LOS, C & 0.901 & 0.528 & 0.128 \\
\hline Seror et al. [64] & Sacrocolpopexy & PRO & 67 & 20 & 47 & dVSS & $\mathrm{BL}, \mathrm{LOS}, \mathrm{C}$ & 0.839 & 0.454 & 0.114 \\
\hline Anger et al. [14] & Sacrocolpopexy & RCT & 78 & 40 & 38 & dVSS & $\mathrm{BL}, \mathrm{C}$ & 0.937 & 0.587 & 0.141 \\
\hline El Hachem et al. [65] & $\begin{array}{l}\text { Various } \\
\text { gynaecological- } \\
\text { unspecified }\end{array}$ & PRO & 91 & 39 & 52 & dVSS & BL, LOS, C & 0.962 & 0.646 & 0.154 \\
\hline Kolvenbach et al. [103] & AAA repair & PRO & 39 & 8 & 31 & ZRSS & LOS & 0.502 & 0.233 & 0.078 \\
\hline Malcolme-Lawes et al. [21] ${ }^{\mathrm{b}}$ & $\begin{array}{l}\mathrm{AF} \text { ablation-robot } \\
30 \mathrm{~s}\end{array}$ & $\mathrm{RCT}$ & 20 & 10 & 10 & Sensei & BT, OT, C & 0.395 & 0.185 & 0.071 \\
\hline Malcolme-Lawes et al. [21] $]^{\mathrm{b}}$ & $\begin{array}{l}\text { AF ablation-robot } \\
60 \mathrm{~s}\end{array}$ & $\mathrm{RCT}^{\mathrm{d}}$ & 20 & 10 & 10 & Sensei & BT, OT, C & 0.395 & 0.185 & 0.071 \\
\hline Steven et al. [83] & AF ablation & RCT & 50 & 25 & 25 & Sensei & OT, $\mathrm{C}$ & 0.791 & 0.410 & 0.107 \\
\hline Kautzner et al. [87] & AF ablation & PRO & 38 & 22 & 16 & Sensei & OT, C & 0.659 & 0.316 & 0.091 \\
\hline Di Biase et al. [86] & AF ablation & PRO & 390 & 193 & 197 & Sensei & OT, C & 1.000 & 0.998 & 0.504 \\
\hline Steven et al. [82] & AF ablation & RCT & 60 & 30 & 30 & Sensei & OT & 0.861 & 0.478 & 0.119 \\
\hline Tilz et al. [22] ${ }^{\mathrm{b}}$ & $\begin{array}{l}\text { AF ablation-robot } \\
30 \mathrm{~W}\end{array}$ & PRO & 29 & 4 & 25 & Sensei & OT & 0.299 & 0.146 & 0.065 \\
\hline Tilz et al. [22] $]^{\mathrm{b}}$ & $\begin{array}{l}\text { AF ablation-robot } \\
20 \mathrm{~W}\end{array}$ & PRO & 35 & 10 & 25 & Sensei & OT & 0.546 & 0.254 & 0.081 \\
\hline Rillig et al. [88] & AF ablation & PRO & 70 & 50 & 20 & Sensei & OT & 0.846 & 0.461 & 0.116 \\
\hline Total & & & 4301 & 1991 & 2310 & & & & & \\
\hline
\end{tabular}

$B L$ blood loss, $B T$ blood transfusion rate, $O T$ skin-to-skin operative (or procedure) time, $L O S$ length of hospital stay, $C 30$-d overall complication rate, $R Y G B$ Roux-en-Y gastric bypass, $C A B G$ coronary artery bypass grafting, $A A A$ abdominal aortic aneurysm, $A F$ atrial fibrillation/flutter, $R C T$ randomised controlled trial, $P R O$ non-randomised prospective comparative study

${ }^{a}$ Relevant to this review

b More than one comparison or study in an article

${ }^{c}$ Gastric banding, implantable gastric stimulator, band revision

${ }^{\mathrm{d}}$ Quasi-RCT (10 patients who underwent robotic AF ablation of 60-s duration were not randomized compared with 10 control patients that were randomised); for Baik 2009, $n=57$ (control) for $\mathrm{C}$ and $n=51$ (control) for OT and LOS, as 6 converted cases were excluded from analysis by authors; for Sarlos 2012, $n=47$ (robotic) and $n=48$ (control) for analysis of C, as no operations were performed in 5 patients, and for BL, OT, and LOS, $n=50$ in each arm, as missing values were replaced with median of available measurements in respective study arm; for Mierdl 2005, $n=30$ (robotic) for analysis of C but $n=24$ for OT, as data not shown for 6 patients

in the robotic arm to be $50.5 \%$ of that in the OS arm (Fig. 4). This reduction was significant $(95 \%$ CI $0.408-0.602)$. There was high heterogeneity in the results $\left(I^{2}=98.0 \%\right)$. Sensitivity analysis on RCTs showed reduction in blood loss, but this was no longer significant (pooled RoM: 0.807, $95 \%$ CI $0.563-1.051, I^{2}=96.3 \%$ ).

\section{Robotic versus MIS}

Twenty-two studies reported blood loss as an outcome measure. Of these, six were RCT studies $[14,50-54]$ and 16 were prospective studies $[16,17,20,55-67]$. Metaanalysis of these studies confirmed a significant reduction in blood loss in favour of robotic surgery, which was
$85.3 \%$ of that experienced by patients in the MIS arm (95\% CI 0.736-0.969) (Fig. 4). The heterogeneity was high $\left(I^{2}=98.2 \%\right)$. Sensitivity analysis performed on RCTs and, however, revealed a non-robust result (pooled RoM: $0.830,95 \%$ CI $0.653-1.008, I^{2}=95.9 \%$ ).

(ii) Blood transfusion rate

\section{Robotic versus $O S$}

Blood transfusion rate was investigated in two RCT [26, 27] and 16 prospective $[17,19,31-36,38,42,45,49,68-$ 71] studies. Forty-two of 2127 patients $(2.0 \%)$ in the robotic group needed blood transfusion compared with 249 
Fig. 3 Risk of bias graphs of randomised controlled trials comparing robotic versus open surgery (above) and robotic versus minimally invasive surgery (below)

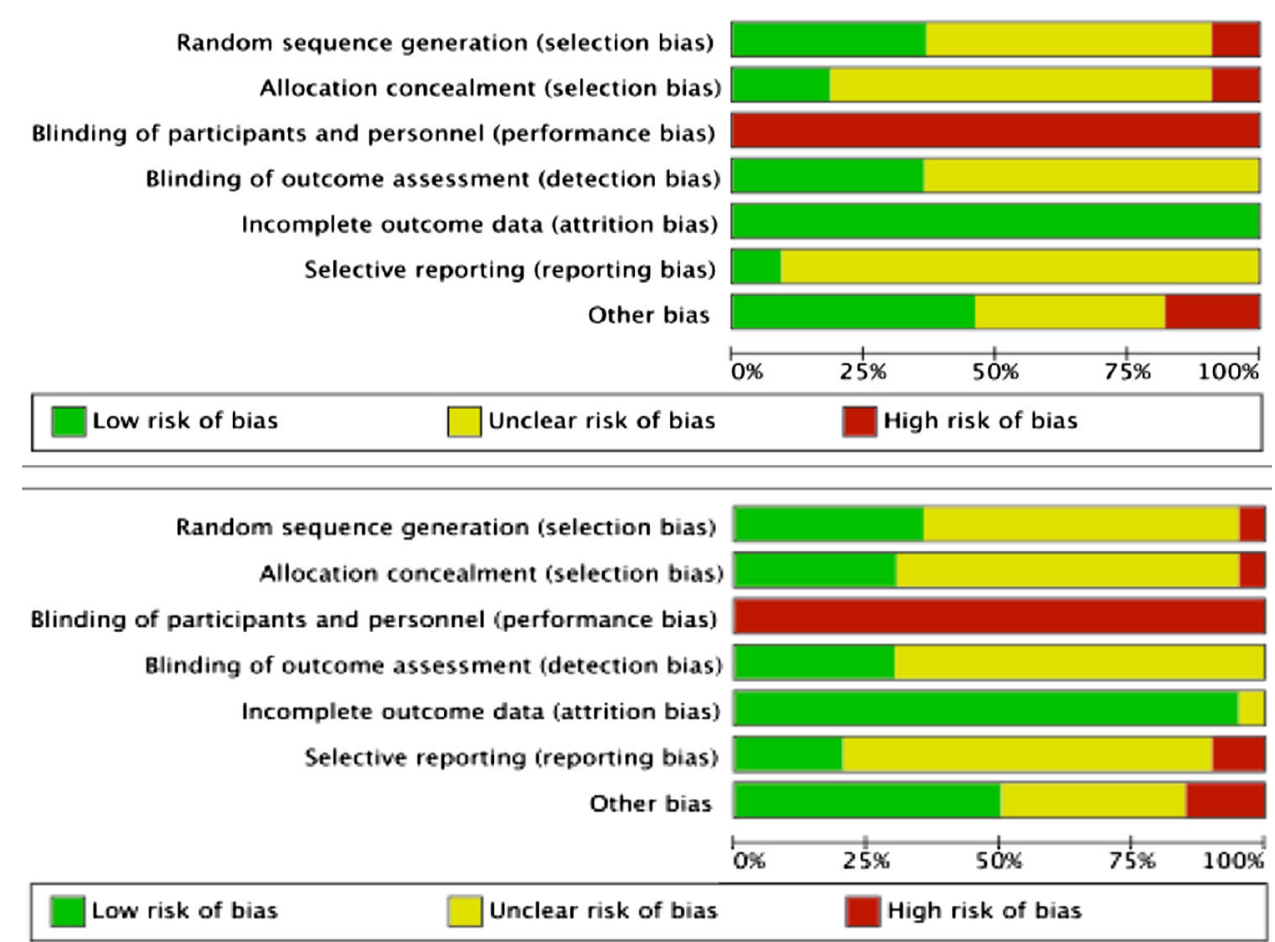

of 1869 patients $(13.3 \%)$ in the open group. One study [27] was excluded from quantitative synthesis, as its effect size was not computable. Meta-analysis of the remaining 17 studies demonstrated the risk of blood transfusion with robotic surgery to be $27.2 \%$ of that of OS. This reduction in favour of robotic surgery was significant $(95 \% \mathrm{CI}$ $0.165-0.449)$. The results showed moderate heterogeneity $\left(I^{2}=55.2 \%\right)$. Sensitivity analysis on RCTs was not done, as only one study was available. In this RCT, no significant difference in blood transfusion requirement was demonstrated (RR 0.800, $95 \%$ CI 0.400-1.600) [26].

\section{Robotic versus MIS}

Six RCT [21, 51, 72-74] and ten prospective [17, 55, 59$63,67,75]$ studies reported blood transfusion requirement. Taking all these studies together, $4.2 \%$ (33/789) of patients who underwent robotic intervention compared with $6.5 \%(56 / 856)$ of MIS patients received blood transfusion. Computation of valid RR was not possible in three studies [21, 51, 63], hence their exclusion from metaanalysis. From the remaining 13 studies, we demonstrated a significant reduction in the requirement for blood transfusion in patients who underwent robotic surgery compared with MIS (pooled RR 0.621, $95 \%$ CI 0.390-0.988). The heterogeneity was low $\left(I^{2}=0.0 \%\right)$. Nevertheless, the result of sensitivity analysis on RCTs was inconsistent (pooled RR 1.329, $95 \%$ CI 0.325-5.438, $I^{2}=0.0 \%$ ).

(iii) Operative time (skin-to-skin)

\section{Robotic versus $O S$}

Sixteen studies assessed operative time. These comprised three RCT [26, 76, 77] and 13 prospective [17, 19, 20, 23, 34, 36, 41, 42, 45-47, 49] studies. Meta-analysis showed robotic surgery to increase operative time by $7.3 \%$, which was significant (95\% CI 1.022-1.124). High heterogeneity was found $\left(I^{2}=91.8 \%\right)$. Sensitivity analysis on RCTs showed a consistent result (pooled RoM: 1.162, 95 \% CI $1.016-1.308, I^{2}=86.8 \%$ ).

\section{Robotic versus MIS}

Operative time was investigated by $12 \operatorname{RCT}[21,50,53,54$, $73,78-83]$ and 18 prospective $[15,17,19,20,22,58,75$, 84-93] studies. There was a significant prolongation of operative time by $13.5 \%$ over MIS when surgical robots were utilised (95\% CI 1.096-1.173). Heterogeneity was high $\left(I^{2}=92.3 \%\right)$. When only RCTs were considered in a sensitivity analysis, the result remained robust (pooled RoM: 1.202, $95 \%$ CI 1.119-1.286, $I^{2}=87.1 \%$ ).

(iv) Length of hospital stay

\section{Robotic versus $O S$}

Thirty studies compared length of hospital stay between robotic and open interventions. There were $4 \mathrm{RCT}[25,26$, $28,77]$ and 26 prospective [16-20, 32, 34-37, 39-43, 45- 


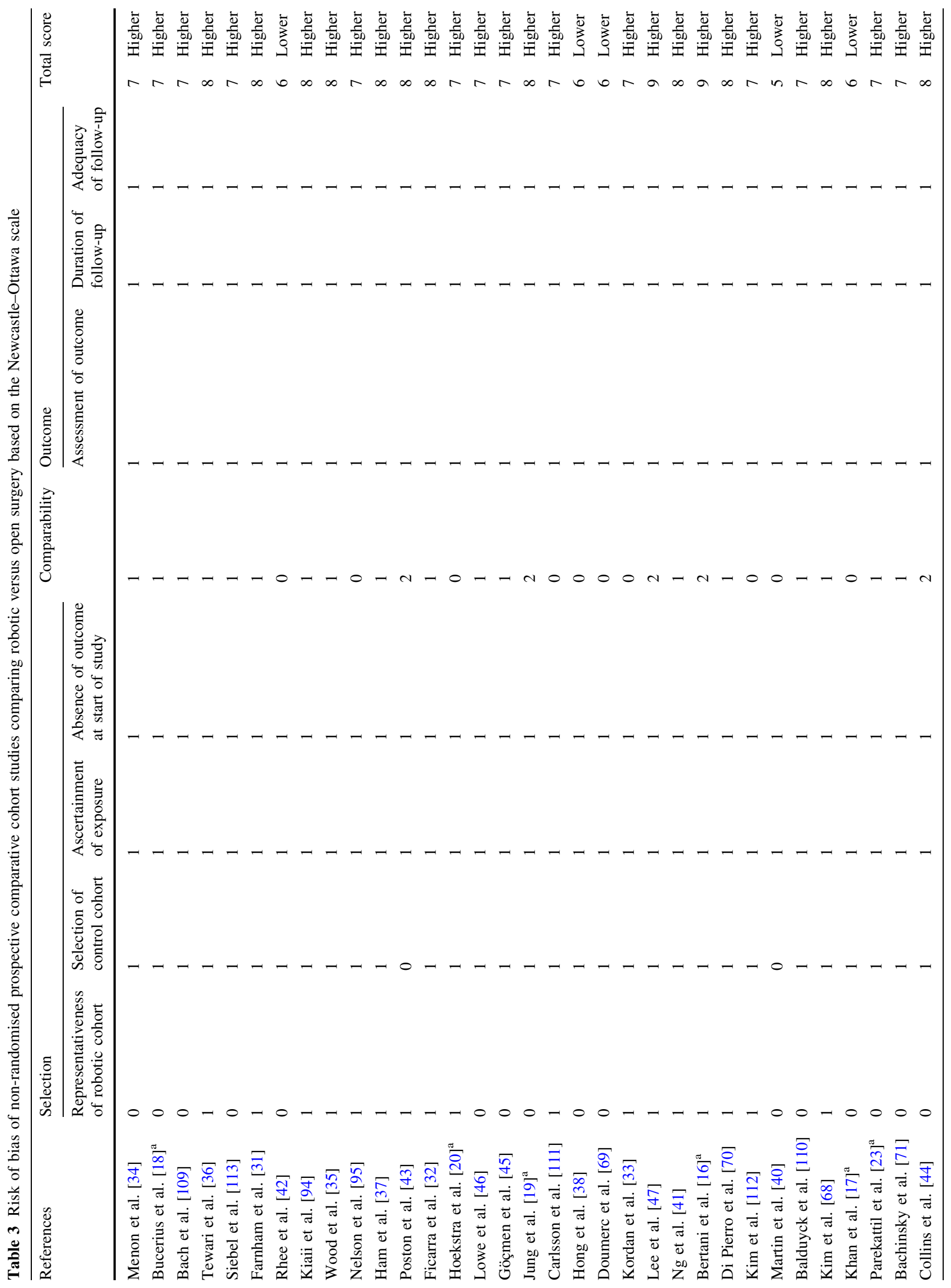




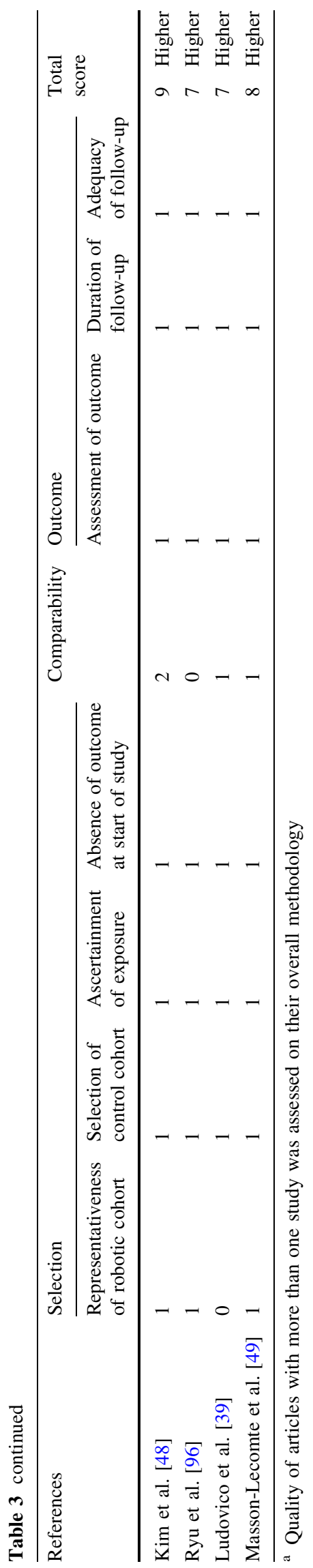

$47,49,68,69,71,94-96]$ studies. The result for one study [26] was not computable. Meta-analysis of the remaining 29 studies revealed length of stay for patients who underwent robotic surgery to be $69.5 \%$ of those who underwent OS. This decrease was significant (95\% CI 0.615-0.774). Heterogeneity was high $\left(I^{2}=98.5 \%\right)$. In contrast, when only RCTs were considered, the improvement in length of stay was lost (pooled RoM: 1.038, 95 \% CI 0.878-1.197, $\left.I^{2}=89.4 \%\right)$.

\section{Robotic versus MIS}

Length of hospital stay was addressed by 40 studies, of which 13 were RCT [50-54, 74, 78, 80, 81, 97-100] and were prospective $[16-20,55-57,59-66,75,85,89,91,101-$ 105] studies. Ten studies $[16,20,50,52,57,91,97,100$, $104,105]$ were excluded from meta-analysis, as their effect sizes were not computable. Meta-analysis of the remaining 30 studies showed no significant difference in duration of stay (pooled RoM: 0.982, $95 \%$ CI 0.936-1.027). High heterogeneity was noted $\left(I^{2}=93.4 \%\right)$. Sensitivity analysis on RCTs remained robust (pooled RoM: $1.001,95 \%$ CI $\left.0.955-1.047, I^{2}=80.2 \%\right)$.

(v) Overall complication rate (30 day)

\section{Robotic versus $O S$}

Overall complications were compared in nine RCT [25, 26, $28-30,76,106-108]$ and 28 prospective $[16,17,19,20,32$, 34-37, 39, 41, 43, 45-47, 49, 68-70, 94-96, 109-113] studies. From these studies, the overall complication rate was $11.6 \%(515 / 4453)$ in the robotic arm compared with $21.4 \%(693 / 3245)$ in the open arm. Results from three studies [29, 96, 109] did not allow for computable RRs. From the remaining 34 studies, meta-analysis demonstrated a significant decrease in overall complication rate in favour of robotic surgery, which was $63.7 \%$ of that with OS (95\% CI 0.483-0.838). High heterogeneity was present $\left(I^{2}=81.9 \%\right)$. Sensitivity analysis on RCTs was, however, inconsistent with the primary analysis (pooled RR 1.090, $95 \%$ CI $0.631-1.881, I^{2}=59.9 \%$ ).

\section{Robotic versus MIS}

Forty-eight studies investigated complications. There were 18 RCT $[14,21,50-54,72,74,78,80,81,83,97-100]$ and 30 prospective $[15-17,19,20,55-66,75,84-87,89,91$, $92,101,104,105]$ studies. Taking all these studies into consideration, the overall complication rate in the robotic arm was $16.1 \%$ (288/1789) compared with $15.7 \%$ (317/ 2025) in the MIS arm. Valid effect sizes in the form of RR were not producible from results of nine studies $[15,52,66$, 


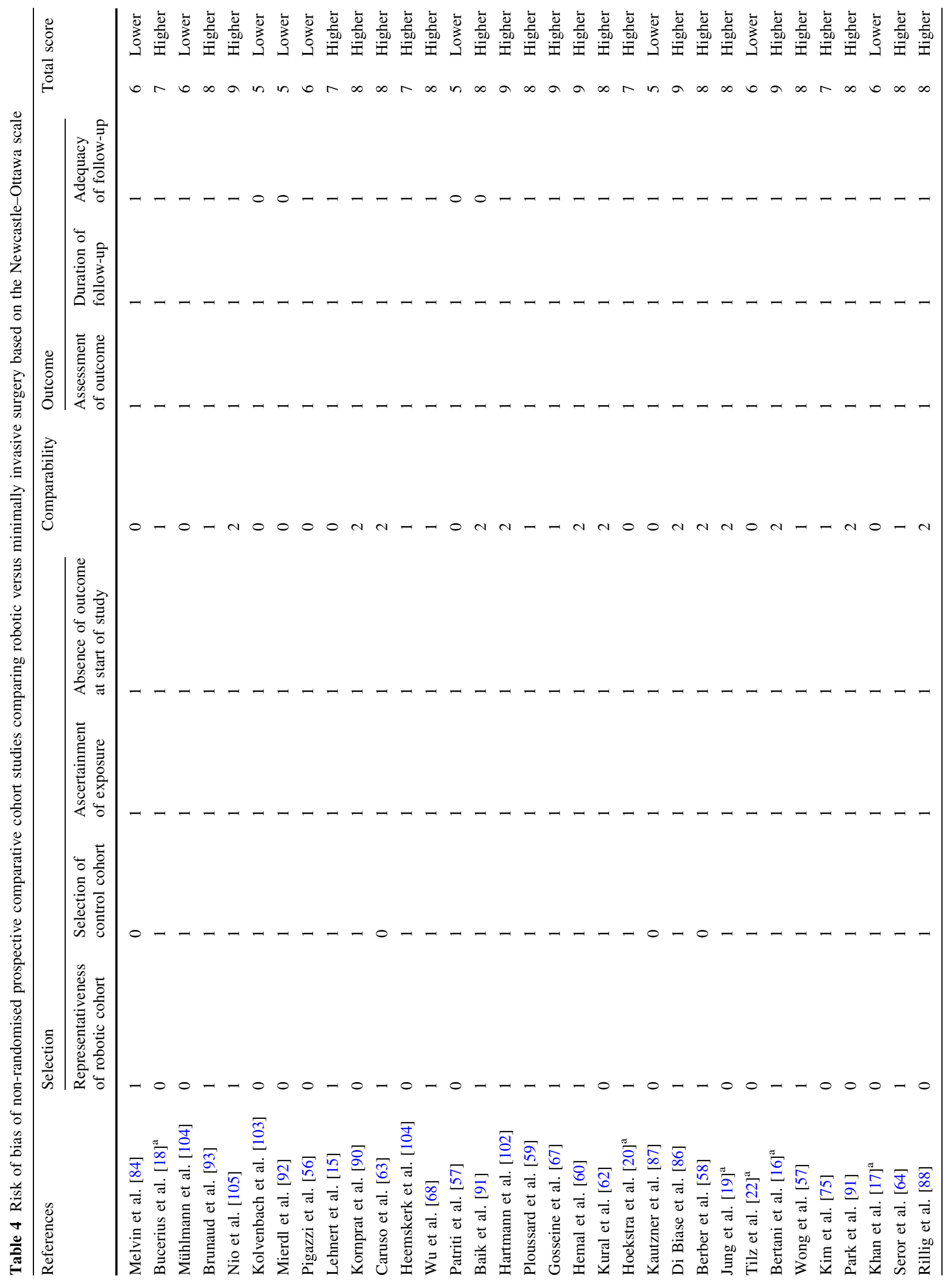




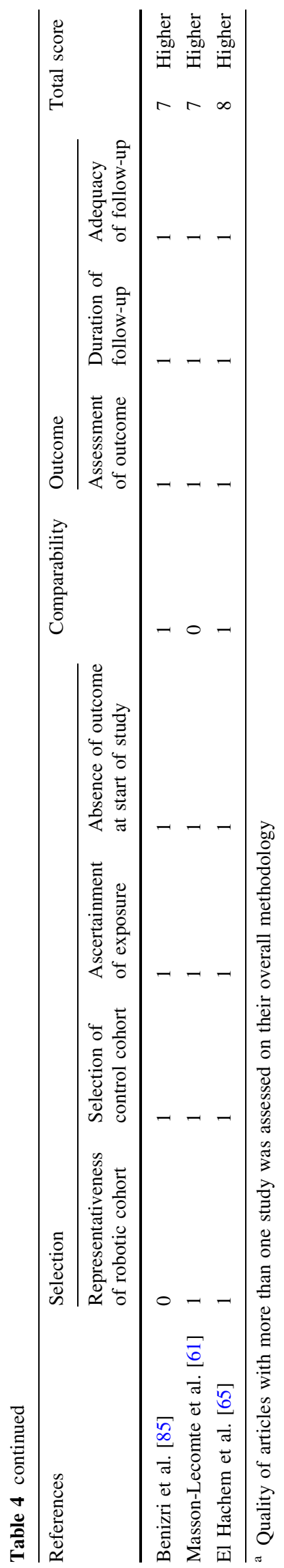

Fig. 4 Forest plots of blood loss; robotic versus open surgery (above), robotic versus minimally invasive surgery (below)

78, 83, 84, 87, 104, 105]. Meta-analysis involving the remaining 39 studies demonstrated no significant difference in overall complication rate between robotic and MIS (pooled RR 0.988, $95 \%$ CI 0.822-1.188). Heterogeneity was low $\left(I^{2}=23.0 \%\right)$. When sensitivity analysis was performed on RCTs, the result remained robust (pooled RR 1.187, $95 \%$ CI $0.851-1.654, I^{2}=15.4 \%$ ).

Results of our meta-analyses are summarised in Fig. 5.

\section{Post hoc power analyses}

With respect to RCT studies, for large effect sizes, just 17 $[14,27-30,51,53,54,72-74,76,77,80,82,107,108]$ of 32 studies $(53.1 \%$ ) had adequate statistical power (that is, power $>80 \%)$. This fell to four studies [27, 28, 76, 107] $(12.5 \%)$ for medium effect sizes. For small effect sizes, no RCT study had adequate power.

Analysis of the 76 prospective studies revealed that just 47 [16-20, 23, 31-37, 39, 41, 43, 47, 49, 55, 57, 59, 61, 64, $65,67-71,75,85,86,88,89,91,94-96,101,111-113]$ of them $(61.8 \%)$ had adequate power for outcome evaluation, assuming large effect sizes. For medium effect sizes, 20 studies [31-33, 35-37, 41, 43, 59, 61, 67-70, 75, 85, $86,95,111,112](26 \cdot 3 \%)$ were sufficiently powered. Only three studies [33, 95, 111] $(4.2 \%)$ had adequate power for small effect sizes.

The lack of statistical power in many studies is not surprising given that in only $16 \mathrm{RCT}(50 \%)$ and six prospective $(7.9 \%)$ studies were primary outcomes clearly defined and a priori power analysis performed (Table 5). Furthermore, only a handful of these studies $[51,54,73,80,82,85]$ were powered to the outcomes investigated in this review.

Results of post hoc power analyses for individual studies are presented in Tables 1 and 2 .

\section{Discussion}

The term "disruptive innovation" represents a process where a product establishes itself at the bottom of a market and climbs through this sector to displace competitors [114]. Initial characteristics of a disruptive innovation model include: (i) simpler products and services, (ii) smaller target markets, and (iii) lower gross margins. As a result, these innovations can "create space" at the bottom of the market to allow new disruptive competitors to emerge. Currently in the field of robotic surgery, the promise of simplicity has yet to be translated into daily practice. Furthermore, the evidence regarding cost efficacy 
Bargar 1998 (Total hip arthroplasty)

Menon 2002 (Prostatectomy)

Tewari 2003 (Prostatectomy)

Farnham 2006 (Prostatectomy)

Nishihara 2006 (Total hip arthroplasty)

Phee 2006 (Cystectomy)

Wood 2007 (Prostatectomy)

Ham 2008 (Prostatectomy)

Poston 2008 (CABG)

Ficarra 2009 (Prostatectomy)

Hoekstra 2009 (Endometrial cancer staging)

Lowe 2009 (Hysterectomy - cervical cancer)

Gömen 2010 (Endometrial cancer staging)

Hong 2010 (Prostatectomy)

Kordan 2010 (Prostatectomy)

Lee 2010 (Thyroidectomy

$\mathrm{Ng} 2010$ (Cystectomy)

Nix 2010 (Cystectomy)

Bertani 2011 (Colectomy)

Bertani 2011 (Rectal resection)

Martin 2011 (Cystectomy)

Song 2011 (Total knee arthroplasty)

Collins 2012 (Sacrocolpopexy)

Khan 2012 (Cystectomy)

Kim 2013 (Thyroidectomy)

Ludovico 2013 (Prostatectomy)

Masson-Lecomte 2013 (Nephrectomy)

Parekh 2013 (Cystectomy)

Song 2013 (Total knee arthroplasty)

Overall (I-squared $=98.0 \%, p=0.000$ )

NOTE: Weights are from random effects analysis

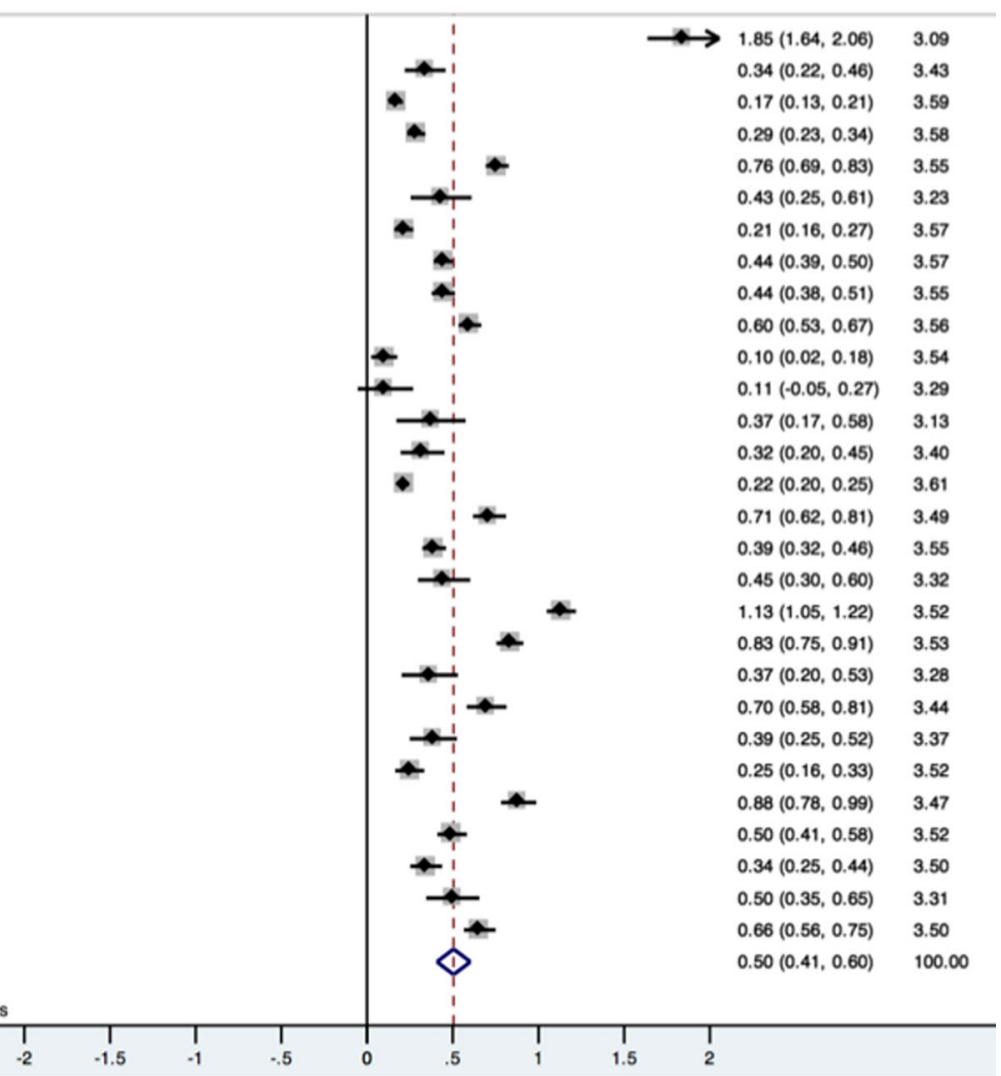

Sedy

seros

Plowes ano6 (Roctal resoction)

Draaisma 2006 (Fundopication)

Zhou 2006 (Chdiocystoctiomy)

Coneso 2006 (Neproctiony)

Wu zocos (Adrentectiony)

Paris zoco (Rectal resection)

Plousserd 2009 (Rrostatectiony)

Gosseine 2009 (Prostactiony)

Hemal 2009 (Neptrectomy)

Kural 2009 (Neptrectomy)

Hoekstra 2009 (Endometrial cancer stagina)

Berber 2010 (Liver resoction)

Bertari 2011 (Coloctomy)

Wong 2011 (Rectopery)

Park 2012 (Colectiony)

Kren 2012 (Cystoctomy)

Strice 2012 (Hysterectomy - berign dseces)

Serco 2012 (Secrocodpopexy)

Porpigla 2013 (Prostatoctomy)

Masson-Lecomte 2013 (Nephrectiony)

B Hachem 2013 (Various graee - unspocifoc)

Anger 2014 (Secrocolipepexy)

Overall (1-squed $=982 \%, p=0.000)$

NOTE: Weigts are trom random elfects andysis

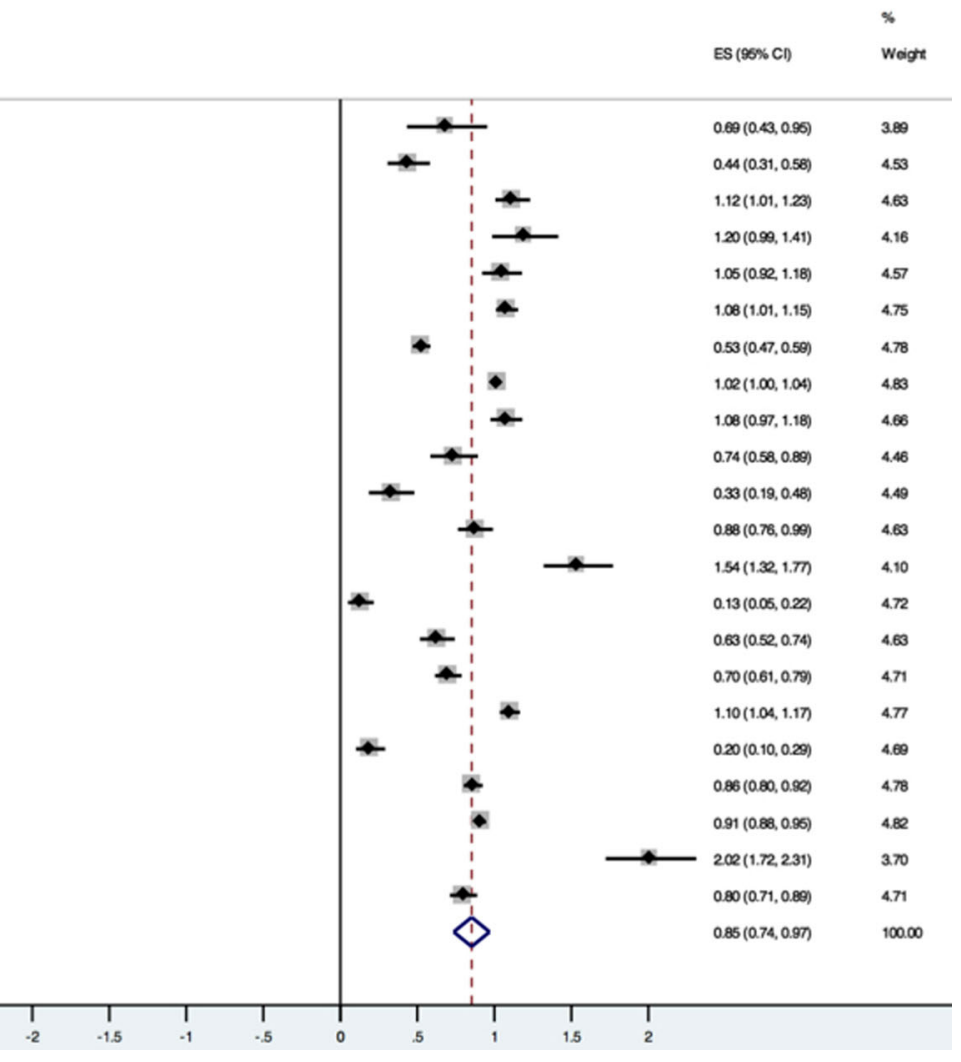


Fig. 5 Pooled proportional change in perioperative outcomes for robotic versus open surgery and robotic versus minimally invasive surgery, with $95 \%$ confidence interval. $R o M$ ratio of means, $R R$ risk ratio, $O S$ open surgery, $M I S$ minimally invasive surgery. *Significant effect

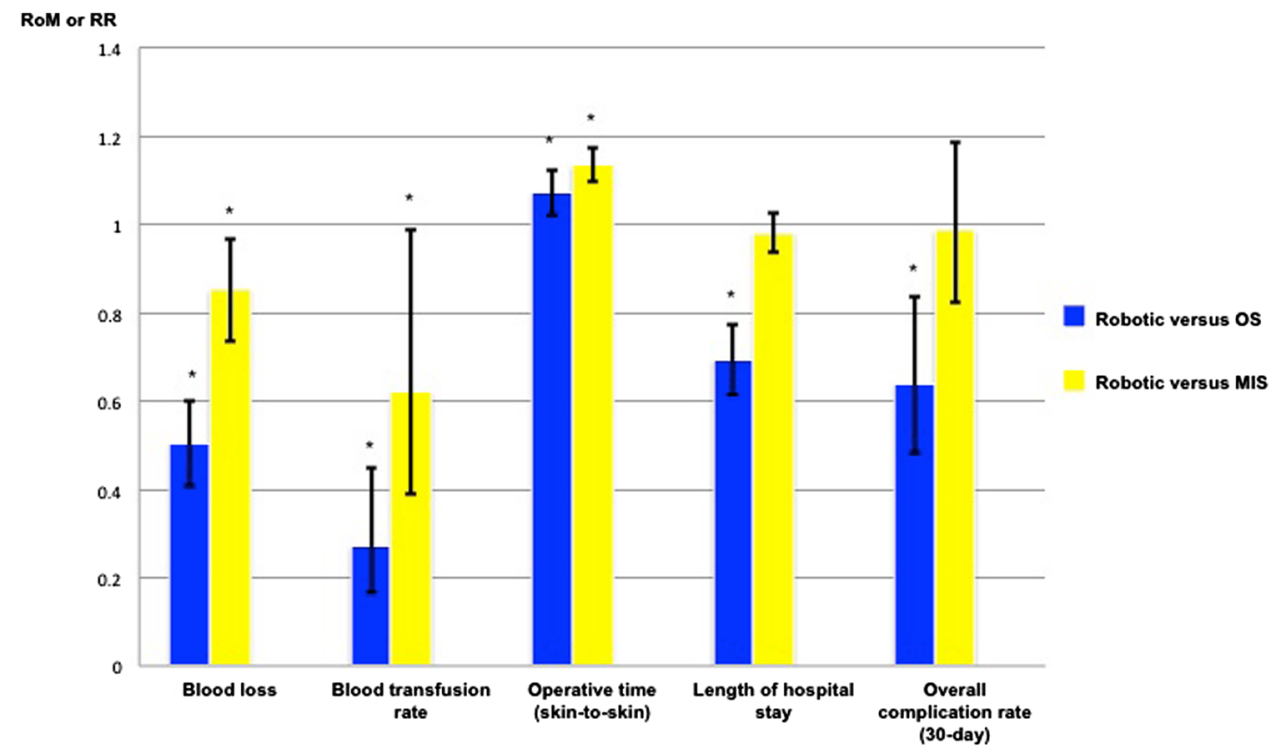

and gross margins has been poorly documented so that decisions regarding the adoption of robotic surgery remain controversial.

However, to disregard robotic surgery completely as an unfulfilled promised in its 30 years of existence may be imbalanced. Our meta-analyses of all RCTs and prospective studies to date, regardless of specialty and procedure type, revealed a decrease in blood loss and blood transfusion rate with robotic surgery when compared with both OS and MIS. Additionally, comparison against OS demonstrated a reduction in length of hospital stay and overall complication rate in favour of robotic surgery.

The ability of robotic surgery to reduce blood loss and need for blood transfusion may be attributed to its advanced features, which could improve surgical precision. This would be important in avoiding injury to vessels and other structures that can cause unintended bleeding. The additional benefits of robotic surgery over OS, in the form of shorter length of hospital stay and fewer complications, may partly be due to its capacity for minimal access. These benefits have been demonstrated in conventional minimally invasive surgical procedures [115-118], where the positive effect of reduced tissue trauma has been implicated [118]. Given its added features, the inability of robotic surgery to achieve improved length of stay and complication rate over MIS can be considered surprising. This may be reflective of the status that surgical robots have not yet exceeded their effects beyond those of conventional minimally invasive platforms for these outcomes. Alternatively, these outcomes may be inadequate markers for accurately capturing the increased precision of robotic surgery. More sensitive assessment tools of precision are advocated in future trials, which might include video appraisal of intraoperative tissue handling, errors, and efficiency [52, 105].
When RCTs were analysed separately, the proportional benefits of robotic surgery were lost. Given their higher level of evidence, these RCTs may be considered as more representative of the true population effect, although they are limited by a profound lack of numbers. We identified only 31 clinical RCTs on robotic surgery, which is a fraction $(0.1 \%)$ of the 28,574 potentially relevant articles. Many RCTs failed to clearly define primary outcomes and perform a priori power analysis, which led to inadequate sample sizes and hence, statistical power necessary for outcome evaluation. Through post hoc analyses, we showed that just over half of all RCTs were adequately powered to detect a true difference in outcomes for large effect sizes. For smaller effect sizes, this deficiency, inevitably, was further amplified. These findings are probably related to common barriers in undertaking successful surgical RCTs, including ethical issues, challenging patient recruitment and randomisation due partly to lack of equipoise, learning curve, inexperience in designing trials, inadequate medical statistical knowledge, problematic long-term follow-up, and insufficient funding and resources [24, 119]. Furthermore, difficulty in blinding is a major methodological barrier [120, 121]. Consequently, all included RCTs were considered to suffer from a high risk of performance bias, and accordingly, a high risk of bias overall [7]. Together, these factors could explain the nonrobust results.

The demonstration of longer operative time with robotic surgery contradicts its proposed aims of facilitating operative tasks that would otherwise be difficult to perform efficiently with conventional tools. One possible explanation is the requirement for additional steps in their deployment. For example, docking is needed for surgical robots such as the dVSS [73, 80]. Hardware issues could also explain the longer operative time, as surgical robotic 
Table 5 Studies with clearly defined primary outcomes and where power analysis was undertaken a priori

\begin{tabular}{|c|c|c|c|c|c|c|c|c|c|}
\hline \multirow[t]{2}{*}{ Study } & \multirow[t]{2}{*}{ Procedure } & \multirow[t]{2}{*}{ Design } & \multicolumn{7}{|c|}{ Primary outcome } \\
\hline & & & OT & LOS & $\mathrm{C}$ & Onc & Func & Cost & Other \\
\hline \multicolumn{10}{|l|}{ Robot versus MIS } \\
\hline Draaisma et al. [50] & Fundoplication & $\mathrm{RCT}$ & & & & & & & $\begin{array}{l}\checkmark \text { Barium swallow, } \\
\text { manometry, ph study }\end{array}$ \\
\hline Morino et al. [78] & Fundoplication & RCT & & & & & & $\checkmark$ & \\
\hline Steven et al. [83] & AF ablation & $\mathrm{RCT}$ & & & & & & & $\boldsymbol{V}$ Radiofrequency duration \\
\hline Steven et al. [82] & AF ablation & RCT & $\checkmark$ & & & & & & \\
\hline $\begin{array}{l}\text { Asimakopoulos et al. } \\
\text { [72] }\end{array}$ & Prostatectomy & RCT & & & & & $\checkmark$ & & \\
\hline Paraiso et al. [80] & Sacrocolpopexy & $\mathrm{RCT}$ & $\checkmark$ & & & & & & \\
\hline Park et al. [51] & Colectomy & $\mathrm{RCT}$ & & $\checkmark$ & & & & & \\
\hline Sarlos et al. [54] & Hysterectomy_benign disease & RCT & $\checkmark$ & & & & & & \\
\hline Porpiglia et al. [53] & Prostatectomy & RCT & & & & & $\checkmark$ & & \\
\hline Paraiso et al. [73] & Hysterectomy_benign disease & RCT & $\checkmark$ & & & & & & \\
\hline Benizri et al. [85] & Roux-en-Y gastric bypass & PRO & & & $\checkmark$ & & & & \\
\hline El Hachem et al. [65] & Various gynae-unspecified & PRO & & & & & & & $\boldsymbol{v}$ Pain \\
\hline Anger et al. [14] & Sacrocolpopexy & $\mathrm{RCT}$ & & & & & & $\checkmark$ & \\
\hline \multicolumn{10}{|l|}{ Robot versus $O S$} \\
\hline Cobb et al. [106] & $\begin{array}{l}\text { Unicompartmental knee } \\
\text { arthroplasty }\end{array}$ & $\mathrm{RCT}$ & & & & & & & $\boldsymbol{V}$ Leg alignment \\
\hline Wood et al. [35] & Prostatectomy & PRO & & & & & & & $\boldsymbol{\sim}$ Quality of life \\
\hline Nix et al. [25] & Cystectomy & $\mathrm{RCT}$ & & & & $\checkmark$ & & & \\
\hline Hong et al. [38] & Prostatectomy & PRO & & & & & & & $\boldsymbol{V}$ Venous gas embolism \\
\hline Song et al. [29] & Total knee arthroplasty & $\mathrm{RCT}$ & & & & & & & $\boldsymbol{V}$ Leg alignment \\
\hline Ringel et al. [77] & Spinal pedicle screw insertion & $\mathrm{RCT}$ & & & & & & & $\boldsymbol{V}$ Implant position \\
\hline Collins et al. [44] & Sacrocolpopexy & PRO & & & & & & & $\begin{array}{l}\mathcal{V} \text { Return to baseline activity } \\
\text { (accelerometer) }\end{array}$ \\
\hline Song et al. [30] & Total knee arthroplasty & $\mathrm{RCT}$ & & & & & & & $\boldsymbol{V}$ Leg alignment \\
\hline Kim et al. [48] & Thyroidectomy & PRO & & & & & & & $\boldsymbol{V}$ Intraocular pressure \\
\hline
\end{tabular}

Note absence of outcome for blood loss and blood transfusion

$O T$ operative time (includes fluoroscopy time), LOS length of stay, $C$ complication, Onc oncological (includes lymph node yield), Func functional (includes erectile function, continence), $R Y G B$ Roux-en-Y gastric bypass, $A F$ atrial flutter/fibrillation, $R C T$ randomised controlled trial, $P R O$ non-randomised prospective comparative study

instruments may be cumbersome to place or switch efficiently, or may be insufficiently adapted for the specific purpose [78, 80, 81, 97].

The surgical learning curve has implications on our findings. Before study commencement, individual surgeons have typically performed far fewer robotic cases than conventional ones [51, 53, 54, 72, 73, 107]. This disparity could disadvantage robotic surgery due to relatively less familiarity. This could further explain the prolonged operative time of robotic surgery. Nevertheless, our demonstration of at least equivalent outcomes for other perioperative variables may be regarded as a favourable effect of robotic surgery. By allowing achievement of similar or better outcomes despite the relative lack of user experience, surgical robots may be important in facilitating training and attainment of competences. Furthermore, many surgeons may view surgical robots as an "enabling technology", without which it would not be possible for them to perform certain complex minimally invasive procedures [122]. Pure laparoscopic radical prostatectomy, which demonstrates significant technical challenges, is an example of a procedure where robotic assistance in suturing and other laparoscopic tasks is important [123]. Although robotic surgery needs to demonstrate more than just equivalent patient outcomes to be cost-effective due to its substantial costs, its potential positive effects on surgeon ability must also be considered.

This systematic review has some limitations. Our focus on blood loss, blood transfusion rate, operative time, length of hospital stay, and complications was based primarily on the fact that these were the most commonly reported 
outcomes in the robotic surgery literature. However, these standard parameters may not fully demonstrate the true value of robotic surgery, especially when the overall benefits are not always clearly perceptible in the short term. Utilisation of dedicated research parameters should be encouraged [124]. Already, there is an increasing inclination towards such parameters that are probably more relevant, including functional, oncological, and quality of life outcomes, specific anatomical-pathological endpoints (such as nerve damage control), and ergonomics. With continuing improvement in outcome parameter selection by clinical research teams, future evidence synthesis centred on these parameters may better reflect the added value of robotic surgery.

Our appraisal of robotic surgery through an exclusively clinical viewpoint has also meant that other elements of innovation evaluation could not be incorporated into our conclusions. These include the impact of surgical robotics on intellectual property and patent generation, resource management, healthcare leadership, mentorship, training, cost efficacy, marketing strategy, business strategy, and stakeholder value generation.

When meta-analyses were possible, the heterogeneity was frequently high. However, this is not unexpected given the wide variability in patient cohorts and interventions. There was additional variability within specific procedures. For instance, Nissen [50, 78, 84, 97-99], Toupet [84], Dor [101], and Thal [15] fundoplications were variant techniques performed in different studies. Furthermore, the extent of robotic assistance varied from its utilisation in anastomotic suturing only [103] to totally robotic procedures $[21,22,82,83,85-88,92,100]$. Methodological diversity in the form of different study designs and risks of bias also contributed to the heterogeneity.

We incorporated different surgical robots in our review, including those that are no longer in use, such as the ZRSS. However, our intention was not to compare outcomes of specific procedures obtainable through currently available robots but to evaluate, via an overview of commonly addressed perioperative outcomes, whether the goals of robotic surgery in general have been achieved. Hence, we offered a unique perspective on robotic surgery by covering the 30 years of its existence. Accordingly, we also elected not to stratify our analysis based on robot or procedure type. Consequently, this restricts the applicability of this review, so that the individual stakeholder interested in outcomes for a specific intervention may not be able to draw sufficiently relevant evidence from our results.

Prospective studies were included to address the paucity of RCTs. Although practical, their inclusion inevitably introduces other biases associated with this study design. Moreover, caution is advised in the interpretation of complication data, as there were inconsistencies in their reporting. Many authors failed to comply with the quality criteria [125] for complication reporting. There was also a lack of agreement in terms of what constitutes complications, such as with regard to blood transfusion and conversion. Nevertheless, this issue is not unique to our included studies [126, 127]. Additionally, studies on robotic surgery continue to suffer from several methodological flaws, including a lack of studies that offer multiple endpoint analysis [128] in such a complex field.

The Society of American Gastrointestinal and Endoscopic Surgeons [122] and European Association of Endoscopic Surgeons [124] consensus statements on robotic surgery have also highlighted the lack of high-quality data in evaluating the health outcomes of this technology. Upcoming research efforts should improve on current methodological deficiencies. The implementation of outcome registries for robotic surgery is important to document and compare benefits and harms and in identifying the direction for future development [122]. More robust controlled trials should be undertaken, particularly in areas where robotic surgery has shown some potential, such as complex hepatobiliary surgery, bariatric and upper gastrointestinal revisional surgery, gastric and oesophageal cancer surgery, rectal surgery, and surgery for large adrenal masses [124].

\section{Conclusions}

After the promising pioneering clinical application of PUMA 560 in 1985 , the stage was set for robotic surgery to assume the role of a significant disruptive innovation in health care. Three decades on, our analysis across a wide range of surgical robots identified their overall positive contribution in reducing blood loss and blood transfusion rate over OS and MIS. Additionally, against OS, they showed overall proportional improvement in length of hospital stay and overall complication rate. These beneficial effects were lost when only RCTs were appraised, although these RCTs were themselves limited. Longer operative time was a common caveat. Further well-conducted surgical trials are needed to confirm these findings. Whilst the barriers for these trials may seem insurmountable, solutions to overcoming them are now increasingly recognised. These may involve ensuring protocol transparency, improving trial dissemination, creating specialised trial units, establishing dedicated outcome monitoring groups, implementing appropriate minimum surgeon experience to reduce the impact of learning curves, and incorporating research training in the surgical curriculum [119]. To ensure better outcomes for future robotic surgery, a multidisciplinary approach during product development involving close collaboration between surgeons and engineers, in addition to inclusive patient engagement, is mandatory. With the advent of more affordable, enriching 
technologies can be modularly incorporated into conventional surgical approaches such as intraoperative fluorescence imaging, high-definition 3-D visualisation, wristed endoscopic hand tools, and navigation systems, robotic surgery risks degenerating into an unfulfilled promise if it fails to innovate in line with stakeholders' needs.

\section{Compliance with ethical standards}

Disclosures Alan Tan, Hutan Ashrafian, Alasdair J. Scott, Sam E. Mason, Leanne Harling, Thanos Athanasiou, and Ara Darzi have no conflicts of interest or financial ties to disclose.

Open Access This article is distributed under the terms of the Creative Commons Attribution 4.0 International License (http://crea tivecommons.org/licenses/by/4.0/), which permits unrestricted use, distribution, and reproduction in any medium, provided you give appropriate credit to the original author(s) and the source, provide a link to the Creative Commons license, and indicate if changes were made.

\section{References}

1. Kwoh YS, Hou J, Jonckheere EA, Hayati S (1988) A robot with improved absolute positioning accuracy for CT guided stereotactic brain surgery. IEEE Trans Biomed Eng 35:153-161

2. Cooper MA, Ibrahim A, Lyu H, Makary MA (2013) Underreporting of robotic surgery complications. J Healthc Qual. doi:10. 1111/jhq. 12036

3. Investor Presentation Q2 (2015) http://investor.intuitivesurgical. com/phoenix.zhtml?c=122359\&p=irol-irhome. Accessed 29 May 2015

4. Yu HY, Hevelone ND, Lipsitz SR, Kowalczyk KJ, Hu JC (2012) Use, costs and comparative effectiveness of robotic assisted, laparoscopic and open urological surgery. J Urol 187:1392-1398

5. Barbash GI, Glied SA (2010) New technology and health care costs - the case of robot-assisted surgery. N Engl J Med 363:701-704

6. Moher D, Liberati A, Tetzlaff J, Altman DG (2009) Preferred reporting items for systematic reviews and meta-analyses: the PRISMA statement. BMJ 339:b2535

7. Higgins J, Green S (2011) Cochrane handbook for systematic reviews of interventions Version 5.1.0 [updated March 2011]. The Cochrane Collaboration. http://www.cochrane-handbook.org

8. Stang A (2010) Critical evaluation of the Newcastle-Ottawa scale for the assessment of the quality of nonrandomized studies in meta-analyses. Eur J Epidemiol 25:603-605

9. Guyatt GH, Oxman AD, Vist G, Kunz R, Brozek J, AlonsoCoello Montori V, Akl EA, Djulbegovic B, Falck-Ytter Y, Norris SL, Williams JA Jr, Atkins D, Meerpohl J, Schunemann HJ (2011) GRADE guidelines: 4. Rating the quality of evidence—study limitations (risk of bias). J Clin Epidemiol 64:407-415

10. Friedrich JO, Adhikari NK, Beyene J (2008) The ratio of means method as an alternative to mean differences for analyzing continuous outcome variables in meta-analysis: a simulation study. BMC Med Res Methodol 8:32

11. DerSimonian R, Laird N (1986) Meta-analysis in clinical trials. Control Clin Trials 7:177-188

12. Higgins JP, Thompson SG, Deeks JJ, Altman DG (2003) Measuring inconsistency in meta-analyses. BMJ 327:557-560
13. Faul F, Erdfelder E, Lang AG, Buchner A (2007) G*Power 3: a flexible statistical power analysis program for the social, behavioral, and biomedical sciences. Behav Res Methods 39:175-191

14. Anger JT, Mueller ER, Tarnay C, Smith B, Stroupe K, Rosenman A, Brubaker L, Bresee C, Kenton K (2014) Robotic compared with laparoscopic sacrocolpopexy: a randomized controlled trial. Obstet Gynecol 123:5-12

15. Lehnert M, Richter B, Beyer PA, Heller K (2006) A prospective study comparing operative time in conventional laparoscopic and robotically assisted Thal semifundoplication in children. J Pediatr Surg 41:1392-1396

16. Bertani E, Chiappa A, Biffi R, Bianchi PP, Radice D, Branchi V, Cenderelli E, Vetrano I, Cenciarelli S, Andreoni B (2011) Assessing appropriateness for elective colorectal cancer surgery: clinical, oncological, and quality-of-life short-term outcomes employing different treatment approaches. Int J Colorectal Dis 26:1317-1327

17. Khan MS, Challacombe B, Elhage O, Rimington P, Coker B, Murphy D, Grieve A, Dasgupta P (2012) A dual-centre, cohort comparison of open, laparoscopic and robotic-assisted radical cystectomy. Int J Clin Pract 66:656-662

18. Bucerius J, Metz S, Walther T, Falk V, Doll N, Noack F, Holzhey D, Diegeler A, Mohr FW (2002) Endoscopic internal thoracic artery dissection leads to significant reduction of pain after minimally invasive direct coronary artery bypass graft surgery. Ann Thorac Surg 73:1180-1184

19. Jung YW, Lee DW, Kim SW, Nam EJ, Kim JH, Kim JW, Kim YT (2010) Robot-assisted staging using three robotic arms for endometrial cancer: comparison to laparoscopy and laparotomy at a single institution. J Surg Oncol 101:116-121

20. Hoekstra AV, Jairam-Thodla A, Rademaker A, Singh DK, Buttin BM, Lurain JR, Schink JC, Lowe MP (2009) The impact of robotics on practice management of endometrial cancer: transitioning from traditional surgery. Int J Med Robot 5:392-397

21. Malcolme-Lawes LC, Lim PB, Koa-Wing M, Whinnett ZI, JamilCopley S, Hayat S, Francis DP, Kojodjojo P, Davies DW, Peters NS, Kanagaratnam P (2013) Robotic assistance and general anaesthesia improve catheter stability and increase signal attenuation during atrial fibrillation ablation. Europace 15:41-47

22. Tilz RR, Chun KR, Metzner A, Burchard A, Wissner E, Koektuerk B, Konstantinidou M, Nuyens D, De Potter T, Neven K, Furnkranz A, Ouyang F, Schmidt B (2010) Unexpected high incidence of esophageal injury following pulmonary vein isolation using robotic navigation. J Cardiovasc Electrophysiol 21:853-858

23. Parekattil SJ, Gudeloglu A, Brahmbhatt J, Wharton J, Priola KB (2012) Robotic assisted versus pure microsurgical vasectomy reversal: technique and prospective database control trial. J Reconstr Microsurg 28:435-444

24. McCulloch P, Taylor I, Sasako M, Lovett B, Griffin D (2002) Randomised trials in surgery: problems and possible solutions. BMJ 324:1448-1451

25. Nix J, Smith A, Kurpad R, Nielsen ME, Wallen EM, Pruthi RS (2010) Prospective randomized controlled trial of robotic versus open radical cystectomy for bladder cancer: perioperative and pathologic results. Eur Urol 57:196-201

26. Parekh DJ, Messer J, Fitzgerald J, Ercole B, Svatek R (2013) Perioperative outcomes and oncologic efficacy from a pilot prospective randomized clinical trial of open versus robotic assisted radical cystectomy. J Urol 189:474-479

27. Nishihara S, Sugano N, Nishii T, Miki H, Nakamura N, Yoshikawa $\mathrm{H}$ (2006) Comparison between hand rasping and robotic milling for stem implantation in cementless total hip arthroplasty. J Arthroplasty 21:957-966 
28. Bargar WL, Bauer A, Borner M (1998) Primary and revision total hip replacement using the Robodoc system. Clin Orthop Relat Res 354:82-91

29. Song EK, Seon JK, Park SJ, Jung WB, Park HW, Lee GW (2011) Simultaneous bilateral total knee arthroplasty with robotic and conventional techniques: a prospective, randomized study. Knee Surg Sports Traumatol Arthrosc 19:1069-1076

30. Song EK, Seon JK, Yim JH, Netravali NA, Bargar WL (2013) Robotic-assisted TKA reduces postoperative alignment outliers and improves gap balance compared to conventional TKA. Clin Orthop Relat Res 471:118-126

31. Farnham SB, Webster TM, Herrell SD, Smith JA Jr (2006) Intraoperative blood loss and transfusion requirements for robotic-assisted radical prostatectomy versus radical retropubic prostatectomy. Urology 67:360-363

32. Ficarra V, Novara G, Fracalanza S, D'Elia C, Secco S, Iafrate M, Cavalleri S, Artibani W (2009) A prospective, non-randomized trial comparing robot-assisted laparoscopic and retropubic radical prostatectomy in one European institution. BJU Int 104:534-539

33. Kordan Y, Barocas DA, Altamar HO, Clark PE, Chang SS, Davis R, Herrell SD, Baumgartner R, Mishra V, Chan RC, Smith JA Jr, Cookson MS (2010) Comparison of transfusion requirements between open and robotic-assisted laparoscopic radical prostatectomy. BJU Int 106:1036-1040

34. Menon M, Tewari A, Baize B, Guillonneau B, Vallancien G (2002) Prospective comparison of radical retropubic prostatectomy and robot-assisted anatomic prostatectomy: the Vattikuti Urology Institute experience. Urology 60:864-868

35. Wood DP, Schulte R, Dunn RL, Hollenbeck BK, Saur R, Wolf JS Jr, Montie JE (2007) Short-term health outcome differences between robotic and conventional radical prostatectomy. Urology 70:945-949

36. Tewari A, Srivasatava A, Menon M (2003) A prospective comparison of radical retropubic and robot-assisted prostatectomy: experience in one institution. BJU Int 92:205-210

37. Ham WS, Park SY, Kim WT, Koo KC, Lee YS, Choi YD (2008) Open versus robotic radical prostatectomy: a prospective analysis based on a single surgeon's experience. J Robot Surg 2:235-241

38. Hong JY, Kim JY, Choi YD, Rha KH, Yoon SJ, Kil HK (2010) Incidence of venous gas embolism during robotic-assisted laparoscopic radical prostatectomy is lower than that during radical retropubic prostatectomy. Br J Anaesth 105:777-781

39. Ludovico GM, Dachille G, Pagliarulo G, D'Elia C, Mondaini N, Gacci M, Detti B, Malossini G, Bartoletti R, Cai T (2013) Bilateral nerve sparing robotic-assisted radical prostatectomy is associated with faster continence recovery but not with erectile function recovery compared with retropubic open prostatectomy: the need for accurate selection of patients. Oncol Rep 29:2445-2450

40. Martin AD, Nunez RN, Castle EP (2011) Robot-assisted radical cystectomy versus open radical cystectomy: a complete cost analysis. Urology 77:621-625

41. Ng CK, Kauffman EC, Lee MM, Otto BJ, Portnoff A, Ehrlich JR, Schwartz MJ, Wang GJ, Scherr DS (2010) A comparison of postoperative complications in open versus robotic cystectomy. Eur Urol 57:274-281

42. Rhee JJ, Lebeau S, Smolkin M, Theodorescu D (2006) Radical cystectomy with ileal conduit diversion: early prospective evaluation of the impact of robotic assistance. BJU Int 98:1059-1063

43. Poston RS, Tran R, Collins M, Reynolds M, Connerney I, Reicher B, Zimrin D, Griffith BP, Bartlett ST (2008) Comparison of economic and patient outcomes with minimally invasive versus traditional off-pump coronary artery bypass grafting techniques. Ann Surg 248:638-646

44. Collins SA, Tulikangas PK, O'Sullivan DM (2012) Effect of surgical approach on physical activity and pain control after sacral colpopexy. Am J Obstet Gynecol 206:438.e1-6

45. Gocmen A, Sanlikan F, Ucar MG (2010) Comparison of roboticassisted surgery outcomes with laparotomy for endometrial cancer staging in Turkey. Arch Gynecol Obstet 282:539-545

46. Lowe MP, Hoekstra AV, Jairam-Thodla A, Singh DK, Buttin BM, Lurain JR, Schink JC (2009) A comparison of robot-assisted and traditional radical hysterectomy for early-stage cervical cancer. J Robot Surg 3:19-23

47. Lee J, Nah KY, Kim RM, Ahn YH, Soh EY, Chung WY (2010) Differences in postoperative outcomes, function, and cosmesis: open versus robotic thyroidectomy. Surg Endosc 24:3186-3194

48. Kim JA, Kim JS, Chang MS, Yoo YK, Dim DK (2013) Influence of carbon dioxide insufflation of the neck on intraocular pressure during robot-assisted endoscopic thyroidectomy: a comparison with open thyroidectomy. Surg Endosc 27:15871593

49. Masson-Lecomte A, Yates DR, Hupertan V, Haertig A, Chartier-Kastler E, Bitker MO, Vaessen C, Roupret M (2013) A prospective comparison of the pathologic and surgical outcomes obtained after elective treatment of renal cell carcinoma by open or robot-assisted partial nephrectomy. Urol Oncol 31:924-929

50. Draaisma WA, Ruurda JP, Scheffer RC, Simmermacher RK, Gooszen HG, Rijnhart-de Jong HG, Busken E, Broeders IA (2006) Randomized clinical trial of standard laparoscopic versus robot-assisted laparoscopic Nissen fundoplication for gastrooesophageal reflux disease. Br J Surg 93:1351-1359

51. Park JS, Choi GS, Park SY, Kim JH, Ryuk JP (2012) Randomized clinical trial of robot-assisted versus standard laparoscopic right colectomy. Br J Surg 99:1219-1226

52. Zhou HX, Guo YH, Yu XF, Bao SY, Liu JL, Zhang Y, Ren YG (2006) Zeus robot-assisted laparoscopic cholecystectomy in comparison with conventional laparoscopic cholecystectomy. Hepatobiliary Pancreat Dis Int 5:115-118

53. Porpiglia F, Morra I, Lucci Chiarissi M, Manfredi M, Mele F, Grande S, Ragni F, Poggio M, Fiori C (2013) Randomised controlled trial comparing laparoscopic and robot-assisted radical prostatectomy. Eur Urol 63:606-614

54. Sarlos D, Kots L, Stevanovic N, von Felten S, Schar G (2012) Robotic compared with conventional laparoscopic hysterectomy: a randomized controlled trial. Obstet Gynecol 120:604611

55. Patriti A, Ceccarelli G, Bartoli A, Spaziani A, Biancafarina A, Casciola L (2009) Short- and medium-term outcome of robotassisted and traditional laparoscopic rectal resection. JSLS 13:176-183

56. Pigazzi A, Ellenhorn JD, Ballantyne GH, Paz IB (2006) Robotic-assisted laparoscopic low anterior resection with total mesorectal excision for rectal cancer. Surg Endosc 20:15211525

57. Wong MT, Meurette G, Rigaud J, Regenet N, Lehur PA (2011) Robotic versus laparoscopic rectopexy for complex rectocele: a prospective comparison of short-term outcomes. Dis Colon Rectum 54:342-346

58. Berber E, Akyildiz HY, Aucejo F, Gunasekaran G, Chalikonda S, Fung J (2010) Robotic versus laparoscopic resection of liver tumours. HPB (Oxford) 12:583-586

59. Ploussard G, Xylinas E, Paul A, Gillion N, Salomon L, Allory Y, Vordos D, Hoznek A, Yiou R, Abbou CC, de la Taille A (2009) Is robot assistance affecting operating room time compared with pure retroperitoneal laparoscopic radical prostatectomy? J Endourol 23:939-943 
60. Hemal AK, Kumar A (2009) A prospective comparison of laparoscopic and robotic radical nephrectomy for T1-2N0M0 renal cell carcinoma. World J Urol 27:89-94

61. Masson-Lecomte A, Bensalah K, Seringe E, Vaessen C, de la Taille A, Doumerc N, Rischmann P, Bruyere F, Soustelle L, Droupy S, Roupret M (2013) A prospective comparison of surgical and pathological outcomes obtained after robot-assisted or pure laparoscopic partial nephrectomy in moderate to complex renal tumours: results from a French multicentre collaborative study. BJU Int 111:256-263

62. Kural AR, Atug F, Tufek I, Akpinar H (2009) Robot-assisted partial nephrectomy versus laparoscopic partial nephrectomy: comparison of outcomes. J Endourol 23:1491-1497

63. Caruso RP, Phillips CK, Kau E, Taneja SS, Stifelman MD (2006) Robot assisted laparoscopic partial nephrectomy: initial experience. J Urol 176:36-39

64. Seror J, Yates DR, Seringe E, Vaessen C, Bitker MO, ChartierKastler E, Roupret M (2012) Prospective comparison of short-term functional outcomes obtained after pure laparoscopic and robotassisted laparoscopic sacrocolpopexy. World J Urol 30:393-398

65. El Hachem L, Acholonu UC Jr, Nezhat FR (2013) Postoperative pain and recovery after conventional laparoscopy compared with robotically assisted laparoscopy. Obstet Gynecol 121:547-553

66. Wu JC, Wu HS, Lin MS, Chou DA, Huang MH (2008) Comparison of robot-assisted laparoscopic adrenalectomy with traditional laparoscopic adrenalectomy-1 year follow-up. Surg Endosc 22:463-466

67. Gosseine PN, Mangin P, Leclers F, Cormier L (2009) Pure laparoscopic versus robotic-assisted laparoscopic radical prostatectomy: comparative study to assess functional urinary outcomes. Prog Urol 19:611-617

68. Kim JC, Yang SS, Jang TY, Kwak JY, Yun MJ, Lim SB (2012) Open versus robot-assisted sphincter-saving operations in rectal cancer patients: techniques and comparison of outcomes between groups of 100 matched patients. Int J Med Robot $8: 468-475$

69. Doumerc N, Yuen C, Savdie R, Rahman MB, Rasiah KK, Pe Benito R, Delprado W, Matthews J, Haynes AM, Stricker PD (2010) Should experienced open prostatic surgeons convert to robotic surgery? The real learning curve for one surgeon over 3 years. BJU Int 106:378-384

70. Di Pierro GB, Baumeister P, Stucki P, Beatrice J, Danuser H, Mattei A (2011) A prospective trial comparing consecutive series of open retropubic and robot-assisted laparoscopic radical prostatectomy in a centre with a limited caseload. Eur Urol 59:1-6

71. Bachinsky WB, Abdelsalam M, Boga G, Kiljanek L, Mumtaz M, McCarty C (2012) Comparative study of same sitting hybrid coronary artery revascularization versus off-pump coronary artery bypass in multivessel coronary artery disease. J Interv Cardiol 25:460-468

72. Asimakopoulos AD, Pereira Fraga CT, Annino F, Pasqualetti P, Calado AA, Mugnier C (2011) Randomized comparison between laparoscopic and robot-assisted nerve-sparing radical prostatectomy. J Sex Med 8:1503-1512

73. Paraiso MF, Ridgeway B, Park AJ, Jelovsek JE, Barber MD, Falcone T, Einarsson JI (2013) A randomized trial comparing conventional and robotically assisted total laparoscopic hysterectomy. Am J Obstet Gynecol 208(368):e1-e7

74. Jimenez Rodriguez RM, Diaz Pavon JM, de La Portilla de Juan F, Prendes Sillero E, Hisnard Cadet Dussort JM, Padillo J (2011) Prospective randomised study: robotic-assisted versus conventional laparoscopic surgery in colorectal cancer resection. Cir Esp 89:432-438

75. Kim YW, Lee HM, Kim NK, Min BS, Lee KY (2012) The learning curve for robot-assisted total mesorectal excision for rectal cancer. Surg Laparosc Endosc Percutan Tech 22:400-405
76. Honl M, Dierk O, Gauck C, Carrero V, Lampe F, Dries S, Quante M, Schwieger K, Hille E, Morlock MM (2003) Comparison of robotic-assisted and manual implantation of a primary total hip replacement. A prospective study. J Bone Joint Surg Am 85-A:1470-1478

77. Ringel F, Stuer C, Reinke A, Preuss A, Behr M, Auer F, Stoffel M, Meyer B (2012) Accuracy of robot-assisted placement of lumbar and sacral pedicle screws: a prospective randomized comparison to conventional freehand screw implantation. Spine (Phila Pa 1976) 37:E496-E501

78. Morino M, Pellegrino L, Giaccone C, Garrone C, Rebecchi F (2006) Randomized clinical trial of robot-assisted versus laparoscopic Nissen fundoplication. Br J Surg 93:553-558

79. Ruurda JP, Visser PL, Broeders IA (2003) Analysis of procedure time in robot-assisted surgery: comparative study in laparoscopic cholecystectomy. Comput Aided Surg 8:24-29

80. Paraiso MF, Jelovsek JE, Frick A, Chen CC, Barber MD (2011) Laparoscopic compared with robotic sacrocolpopexy for vaginal prolapse: a randomized controlled trial. Obstet Gynecol 118:1005-1013

81. Morino M, Beninca G, Giraudo G, Del Genio GM, Rebecchi F, Garrone C (2004) Robot-assisted vs laparoscopic adrenalectomy: a prospective randomized controlled trial. Surg Endosc 18:1742-1746

82. Steven D, Servatius H, Rostock T, Hoffmann B, Drewitz I, Mullerleile K, Sultan A, Aydin MA, Meinertz T, Willems S (2010) Reduced fluoroscopy during atrial fibrillation ablation: benefits of robotic guided navigation. J Cardiovasc Electrophysiol 21:6-12

83. Steven D, Rostock T, Servatius H, Hoffmann B, Drewitz Mullerleile K, Meinertz T, Willems S (2008) Robotic versus conventional ablation for common-type atrial flutter: a prospective randomized trial to evaluate the effectiveness of remote catheter navigation. Heart Rhythm 5:1556-1560

84. Melvin WS, Needleman BJ, Krause KR, Scheneider C, Ellison EC (2002) Computer-enhanced vs. standard laparoscopic antireflux surgery. J Gastrointest Surg 6:11-15 (discussion 15-16)

85. Benizri EI, Renaud M, Reibel N, Germain A, Ziegler O, Zarnegar R, Ayav A, Bresler L, Brunaud L (2013) Perioperative outcomes after totally robotic gastric bypass: a prospective nonrandomized controlled study. Am J Surg 206:145-151

86. Di Biase L, Wang Y, Horton R, Gallinghouse GJ, Mohanty P, Sanchez J, Patel D, Dare M, Canby R, Price LD, Zagrodzky JD, Bailey S, Burkhardt JD, Natale A (2009) Ablation of atrial fibrillation utilizing robotic catheter navigation in comparison to manual navigation and ablation: single-center experience. J Cardiovasc Electrophysiol 20:1328-1835

87. Kautzner J, Peichl P, Cihak R, Wichterle D, Mlcochova $H$ (2009) Early experience with robotic navigation for catheter ablation of paroxysmal atrial fibrillation. Pacing Clin Electrophysiol 32(Suppl 1):S163-S166

88. Rillig A, Meyerfeldt U, Tilz RR, Talazko J, Arya A, Zvereva V, Birkemeyer R, Miljak T, Hajredini B, Wohlmuth P, Fink U, Jung W (2012) Incidence and long-term follow-up of silent cerebral lesions after pulmonary vein isolation using a remote robotic navigation system as compared with manual ablation. Circ Arrhythm Electrophysiol 5:15-21

89. Baik SH, Kwon HY, Kim JS, Hur H, Sohn SK, Cho CH, Kim H (2009) Robotic versus laparoscopic low anterior resection of rectal cancer: short-term outcome of a prospective comparative study. Ann Surg Oncol 16:1480-1487

90. Kornprat P, Werkgartner G, Cerwenka H, Bacher H, El-Shabrawi A, Rehak P, Mischinger HJ (2006) Prospective study comparing standard and robotically assisted laparoscopic cholecystectomy. Langenbecks Arch Surg 391:216-221

91. Park JY, Jo MJ, Nam BH, Kim Y, Eom BW, Yoon HM, Ryu KW, Kim YW, Lee JH (2012) Surgical stress after robot- 
assisted distal gastrectomy and its economic implications. Br J Surg 99:1554-1561

92. Mierdl S, Byhahn C, Lischke V, Aybek T, Wimmer-Greinecker G, Dogan S, Viehmeyer S, Kessler P, Westphal K (2005) Segmental myocardial wall motion during minimally invasive coronary artery bypass grafting using open and endoscopic surgical techniques. Anesth Analg 100:306-314

93. Brunaud L, Bresler L, Ayav A, Tretou S, Cormier L, Klein M, Boissel P (2003) Advantages of using robotic Da Vinci system for unilateral adrenalectomy: early results. Ann Chir 128:530-535

94. Kiaii B, McClure RS, Stitt L, Rayman R, Dobkowski WB, Jablonsky G, Novick RJ, Boyd WD (2006) Prospective angiographic comparison of direct, endoscopic, and telesurgical approaches to harvesting the internal thoracic artery. Ann Thorac Surg 82:624-628

95. Nelson B, Kaufman M, Broughton G, Cookson MS, Chang SS, Herrell SD, Baumgartner RG, Smith JA Jr (2007) Comparison of length of hospital stay between radical retropubic prostatectomy and robotic assisted laparoscopic prostatectomy. J Urol 177:929-931

96. Ryu HR, Lee J, Park JH, Kang SW, Jeong JJ, Hong JY, Chung WY (2013) A comparison of postoperative pain after conventional open thyroidectomy and transaxillary single-incision robotic thyroidectomy: a prospective study. Ann Surg Oncol 20:2279-2284

97. Cadiere GB, Himpens J, Vertruyen M, Bruyns J, Germay O, Leman G, Izizaw R (2001) Evaluation of telesurgical (robotic) NISSEN fundoplication. Surg Endosc 15:918-923

98. Muller-Stich BP, Reiter MA, Wente MN, Bintintan VV, Koninger J, Buchler MW, Gutt CN (2007) Robot-assisted versus conventional laparoscopic fundoplication: short-term outcome of a pilot randomized controlled trial. Surg Endosc 21:1800-1805

99. Nakadi IE, Melot C, Closset J, DeMoor V, Betroune K, Feron P, Lingier P, Gelin M (2006) Evaluation of da Vinci Nissen fundoplication clinical results and cost minimization. World J Surg 30:1050-1054

100. Sanchez BR, Mohr CJ, Morton JM, Safadi BY, Alami RS, Curet MJ (2005) Comparison of totally robotic laparoscopic Roux-enY gastric bypass and traditional laparoscopic Roux-en-Y gastric bypass. Surg Obes Relat Dis 1:549-554

101. Hartmann J, Menenakos C, Ordemann J, Nocon M, Raue W, Braumann C (2009) Long-term results of quality of life after standard laparoscopic vs. robot-assisted laparoscopic fundoplications for gastro-oesophageal reflux disease. A comparative clinical trial. Int J Med Robot 5:32-37

102. Heemskerk J, de Hoog DE, van Gemert WG, Baeten CG, Greve JW, Bouvy ND (2007) Robot-assisted vs. conventional laparoscopic rectopexy for rectal prolapse: a comparative study on costs and time. Dis Colon Rectum 50:1825-1830

103. Kolvenbach R, Schwierz E, Wasilljew S, Miloud A, Puerschel A, Pinter L (2004) Total laparoscopically and robotically assisted aortic aneurysm surgery: a critical evaluation. J Vasc Surg 39:771-776

104. Muhlmann G, Klaus A, Kirchmayr W, Wykypiel H, Unger A, Holler E, Nehoda H, Aigner F, Weiss HG (2003) DaVinci robotic-assisted laparoscopic bariatric surgery: is it justified in a routine setting? Obes Surg 13:848-854

105. Nio D, Bemelman WA, Busch OR, Vrouenraets BC, Gouma DJ (2004) Robot-assisted laparoscopic cholecystectomy versus conventional laparoscopic cholecystectomy: a comparative study. Surg Endosc 18:379-382

106. Cobb J, Henckel J, Gomes P, Harris S, Jakopec M, Rodriguez F, Barrett A, Davies B (2006) Hands-on robotic unicompartmental knee replacement: a prospective, randomised controlled study of the Acrobot system. J Bone Joint Surg Br 88:188-197

107. Nakamura N, Sugano N, Nishii T, Kakimoto A, Miki H (2010) A comparison between robotic-assisted and manual implantation of cementless total hip arthroplasty. Clin Orthop Relat Res 468:1072-1081

108. Park SE, Lee CT (2007) Comparison of robotic-assisted and conventional manual implantation of a primary total knee arthroplasty. J Arthroplasty 22:1054-1059

109. Bach CM, Winter P, Nogler M, Gobel G, Wimmer C, Ogon M (2002) No functional impairment after Robodoc total hip arthroplasty: gait analysis in 25 patients. Acta Orthop Scand 73:386-391

110. Balduyck B, Hendriks JM, Lauwers P, Mercelis R, Ten Broecke P, Van Schil P (2011) Quality of life after anterior mediastinal mass resection: a prospective study comparing open with robotic-assisted thoracoscopic resection. Eur J Cardiothorac Surg 39:543-548

111. Carlsson S, Nilsson AE, Schumacher MC, Jonsson MN, Volz DS, Steineck G, Wiklund PN (2010) Surgery-related complications in 1253 robot-assisted and 485 open retropubic radical prostatectomies at the Karolinska University Hospital, Sweden. Urology 75:1092-1097

112. Kim SC, Song C, Kim W, Kang T, Park J, Jeong IG, Lee S, Cho YM, Ahn H (2011) Factors determining functional outcomes after radical prostatectomy: robot-assisted versus retropubic. Eur Urol 60:413-419

113. Siebel T, Kafer W (2005) Clinical outcome following robotic assisted versus conventional total hip arthroplasty: a controlled and prospective study of seventy-one patients. Z Orthop Ihre Grenzgeb 143:391-398

114. Christensen CM (1997) The innovator's dilemma: when new technologies cause great firms to fail. Harvard Business School Press, Boston

115. Cheng Q, Pang TC, Hollands MJ, Richardson AJ, Pleass H, Johnston ES, Lam VW (2014) Systematic review and metaanalysis of laparoscopic versus open distal gastrectomy. J Gastrointest Surg 18:1087-1099

116. Rondelli F, Trastulli S, Avenia N, Schillaci G, Cirocchi R, Gulla N, Mariani E, Bistoni G, Noya G (2012) Is laparoscopic right colectomy more effective than open resection? A meta-analysis of randomized and nonrandomized studies. Colorectal Dis 14:e447-e469

117. Tang K, Li H, Xia D, Hu Z, Zhuang Q, Liu J, Xu H, Ye Z (2014) Laparoscopic versus open radical cystectomy in bladder cancer: a systematic review and meta-analysis of comparative studies. PLoS ONE 9:e95667

118. Vennix S, Pelzers L, Bouvy N, Beets GL, Pierie JP, Wiggers T, Breukink S (2014) Laparoscopic versus open total mesorectal excision for rectal cancer. Cochrane Database Syst Rev 4:Cd005200

119. Garas G, Ibrahim A, Ashrafian H, Ahmed K, Patel V, Okabayashi K, Skapinakis P, Darzi A, Athanasiou T (2012) Evidence-based surgery: barriers, solutions, and the role of evidence synthesis. World J Surg 36:1723-1731

120. Lilford R, Braunholtz D, Harris J, Gill T (2004) Trials in surgery. Br J Surg 91:6-16

121. Stirrat GM, Farrow SC, Farndon J, Dwyer N (1992) The challenge of evaluating surgical procedures. Ann R Coll Surg Engl 74:80-84

122. Herron DM, Marohn M (2008) A consensus document on robotic surgery. Surg Endosc 22:313-325 (discussion 311-312)

123. Harrell AG, Heniford BT (2005) Minimally invasive abdominal surgery: lux et veritas past, present, and future. Am J Surg 190:239-243

124. Szold A, Bergamaschi R, Broeders I, Dankelman J, Forgione A, Lango T, Melzer A, Mintz Y, Morales-Conde S, Rhodes M, Satava R, Tang CN, Vilallonga R (2015) European Association of Endoscopic Surgeons (EAES) consensus statement on the use of robotics in general surgery. Surg Endosc 29:253-288

125. Martin RC 2nd, Brennan MF, Jaques DP (2002) Quality of complication reporting in the surgical literature. Ann Surg 235:803-813 
126. Haidich AB, Birtsou C, Dardavessis T, Tirodimos I, Arvanitidou M (2011) The quality of safety reporting in trials is still suboptimal: survey of major general medical journals. J Clin Epidemiol 64:124-135

127. Ioannidis JP, Lau $\mathbf{J}$ (2001) Completeness of safety reporting in randomized trials: an evaluation of 7 medical areas. JAMA 285:437-443
128. Almeida GM, Duarte TL, Farmer PB, Steward WP, Jones GD (2008) Multiple end-point analysis reveals cisplatin damage tolerance to be a chemoresistance mechanism in a NSCLC model: implications for predictive testing. Int $\mathrm{J}$ Cancer 122:1810-1819 